Aus dem Institut für pathologische Anatomie an der Universität

Parma (Direktor: Prof. Dr. Pietro Guizzetti).

\title{
Ein Fall von Splenomegalie.
}

(Histologische und klinische Untersuchungen.)

Von Dr. Giacomo Zaccarini,

Ex-Adjutant der path. Anatomie und Hilfschirurg am Hospital zu Bergamo (Italien).

(Mit 4 Abbildungen)

Ich habe einige histologische Untersuchungen an einer splenomegalischen, in unserem Hospital entfernten Milz vorgenommen. Die Patientin erlag der Operation und ich versuchte, die gemachten Beobachtungen mit den aus der Autopsie sich ergebenden zu vergleichen.

Da es sich um eine Frau handelte, die während ihres Lebens ein ziemlich interessantes Syndrom, Splenomegalie und Anämie zeigte, war dieser Fall klinisch wie auch anatomisch untersuchungswert. Ich habe mich aber nur auf die Untersuchungen anatomisch-histologischen Charakters beschränkt, welche sich zum Studium der Natur dieser Krankheit am besten eigneten und vielleicht mehr als die klinischen Befunde. Es sind daher die unten angeführten klinischen Mitteilungen sehr kurz.

Es handelte sich um eine etwa 40 jährige Frau ohne besondere vorhergehende Erscheinungen. Seit 5 Jahren hatte sie eine langsam verlaufende Splenomegalie. Die Milz ragte bei der Untersuchung mehr als $5 \mathrm{~cm}$ über den Rippenbogen, und richtete sich gegen die Medianlinie. Es bestand gleichzeitig eine ausgesprochene Anämie, die sich sekundär der splenischen Anschwellung beigesellt hatte.

Dic Blutuntersuchung ergab 3500000 rote Blutkörperchen und 35 Proz. Hámoglobin. Die Globularmasse hatte an Wert verloren. Man fand nichts vor, was das hamatologische Bild veränderte. Die Leber hatte an Volumen nicht zugenommen. Der Harn war etwas karg und enthielt keine pathologischen Elemente. Mäßige Menge freier Flüssigkeit im Bauche. 
Die Diagnose war auf primäre Splenomegalie gestellt neben Anamie, wahrscheinlich auf Bantısche Krankheit beruhend. Es sollen hier nicht die Gründe erwähnt werden, welche bei dieser Krankheit zur Vornahme der Operation fuhrten. Auf Grund der von Banti und $\mathrm{Colzi}$ aufgestellten und von einer langen Reihe Chirurgen bestätigten Anschauungen, wurde diese Frau von Prof. Gar b a rini am 9. XI. 1910 durch Splenektomie operiert. Die Operation bestätigte, daß ein mảßiger, klarer Flüssigkeitserguß im Bauche bestand und daß die Leber sich in ihren normalen Grenzen hielt. Der postoperative Verlauf war in den ersten 13 Tagen apyretisch, obwohl der Zustand der Patientin immer schwer blieb. Dann trat ein starkes Fieber (39 Grad) ohne Zitterbewegungen auf, am I5. Tage Darmblutfluß und am nachsten Tage der Tod.

Dic Milz wog $1800 \mathrm{~g}$. Die Kapsel war fast normal, sehr gespannt und zeigte keine außergewohnliche Adhäsion. Am Hilus ist ein großes schlaffes Gefaß (Transversaldurchmesser $7 \mathrm{~cm}$ ), das eine $V$ ene zu sein scheint. Sie hat eine weißliche, unregelmäßig runzlige Innenfläche, auf der hier und da weiße Plattchen, wie bei der Kalkinfiltration, zerstreut sind. Um diese splenische Vene ist ein pankreashaltiger Muff, der ein normales Ausschen hat. Man kann ferner am Milzhilus andere venöse Gefaße sehen, die mit wcißlichen, unregelmaßig verdickten, sklerotischen Wänden - aber ohne Kalkinfiltration - versehen sind und in die Milz hineintreten.

Auf der Schnittflache hat die Milz eine rote Farbe, ähnlich dem Muskel eines jungen Ochsen, aber etwas verblichen. Die Fläche ist dicht, kontinuierlich, ziemlich trocken. Unter dem Fingerdrucke läßt sie sich einbrechen, indem sie Widerstand we eine Niere leistet. Auf der Schnittfläche ist die Kapsel nicht verdickt und strahlt keine Balken aus. Die Venen sind gut sichtbar, mit einem offenen Lumen und einer etwas verdickten Wand in Form eines weißlichen Ringes, der bei den mittleren Adern eine Dicke ron $1 / 4-1 / 2 \mathrm{~mm}$ erreicht. Gegen den unteren Pol ist unter der Kapsel en kegelförmıger, weißlicher, schlecht begrenzter Platz, der als ein anamischer in Entwicklung stehender Infarkt gedeutet werden kann. Stellenweise sieht man in der Pulpa hämorrhagische Flecken. Im großen Ganzen hat man den Eindruck einer ausgedehnten, bindegewcbigen Milzhyperplasie nebst Verdickung der Venenwande, aber ohne Anteilnahme der Kapsel und ohne daß wir die Follkeln sehen können. Durch Jod bekommt man keine Anzcichen von Amyloidosis.

Die Sektion wurde am 25. XI. I9Io ausgeführt. Esterina Dazzi.) - Schlechtgenährte Leichc. Regelmäßiges Skelett. Mediane Laparotomiewunde, $20 \mathrm{~cm}$ lang, vollkommen vernarbt. Der Schädel wird nicht geöffnet. Die r e chte Lunge ist frei und hat nur eine leichte Basalverwachsung. Der obere und mittlere Lappen sind gedunsen und anämisch. Der untere Lappen ist ziemlich angedrängt 
und sehr ödematös. Die Bronchien sind anämisch und wenig schleimig. Pulsadern und Lungenacini normal. Die linke Lunge ist an dem vorderen Rand emphysematös. Oberer Lungenlappen gedunsen, anämisch und emphysematös. Der untere Lungenlappen ebenfalls gedunsen, etwas angedrängt und ödematös. Bronchien, Lungengefäße und Lungenacini normal. Trachea mit wenig Schleim bedeckt und anämisch. Es fehlt dabei jede Stauung in den Drusen an der Bifurkation und am Hilus der Trachea. Brusta o $\mathrm{ta}$ ziemlich klein, mit glatter normaler Wand. Das $\mathrm{Herz}$ hat wenige Milchflecke auf dem Epikardium. Fett reichlich vorhanden. Es besteht ferner eine sehr kleine Öffnung des Botallischen Loches. Links kontrahiert, transversal $=\mathrm{I} 2 \mathrm{~cm}$ und Vertikaldurchmesser $9^{1 / 2} \mathrm{~cm}$. Linker Herzventrikel $16 \mathrm{~mm}$, rechter $6 \mathrm{~mm}$. Weder Insuffizienz noch Stenose. Herzklappen normal, ohne Atherom oder hyaline Knoten. Myokard sehr anämisch, ohne scheinbare Fettstreifen. Endokard normal. Herzvenen ebenfalls normal. Der $\mathrm{M}$ a g e $\mathrm{n}$ ist klein, mit einer anämischen, am Fundus dünnen Schleimhaut, ohne Autodigestion. Im Netzbeutel gegen den Schwanz des Pankreas ist eine faustgroße, aus einer klebrigen Flüssigkeit bestehenden Ansammlung (Pankreassaft?) mit einigen Flocken vorhanden. Der Pylorus ist normal. Das D u od en u m ist durch Gallenfarbstoff gefärbt, die Schleimhaut normal. Das M esenterium ist sehr fettreich und zeigt zahlrciche Stränge, die von der Mesenteriumwurzel ausgehend sich nach dem Dunndarm richten; offenbar stellen sie Venen dar. Das $\mathrm{Pankreas}$ hat eine normale Länge und ist von zahlreichen Fettläppchen durchsetzt. Die splen ische $\mathrm{V}$ ene ist ungeheuer ausgedehnt gegen die Mitte zu. Diese Ausdehnung ist eine fortschreitende, sie beginnt am Schwanze und nimmt allmählich zu. An ihrer höchsten Ausdehnungsstelle mißt ihr Durchmesser etwa $10 \mathrm{~cm}$. Die Vene ist durch einen großen mehr oder weniger anhaftenden Thrombus gänzlich verstopft. Dieser 'Thrombus ist teils rot, teils weiß und hat große Verfettungszentren. Die Wandung dieser Vene ist außerordentlich verdickt und gleicht jener einer atheromatösen Aorta. Ihre Dicke ist $4 \mathrm{~mm}$. Sie ist durch zahlreiche bindegewebige Streifen und durch gelbe kalkartige Stellen gekennzeichnet.

Dieselben Veränderungen, aber etwas weniger ausgesprochen, haben wir in der V. porta, die ebenfalls außergewöhnlich angeschwollen ist, derart, daß sie sich als ein globulärer, mandaringroßer Korper mit $8 \mathrm{~cm}$ Durchmesser zeigt. Die Venenwand hat ebenfalls das Aussehen einer ubermäßig verdickten Arterie und enthält einen großen, an vielen Stellen anhaftenden Thrombus, der auch die oben beschriebenen Charaktere besitzt.

Die Wandausdehnung und die -verdickung der V. porta besteht durch alle intrahepatischen Äste derselben fort; letztere in toto 
durch kanalikuläre, mehr oder weniger anhaftende, teils rote, teils weiße Thrombi verstopft, welche dieselben Charaktere wie die oben erwähnten zeigen.

Solche Thrombosen schreiten bis an die feinsten Verästelungen der V. porta fort; die Gallenkanäle und die intrahepatischen Gallengange sind dagegen normal, und normal sind auch der Stamm der hepatischen Arterie und die intrahepatischen Äste derselben.

Die Leber hat leicht an Volumen abgenommen und besitzt einen schmalen Rand. Die Abnahme hat hauptsächlich den rechten Leberlappen betroffen; der linke Leberlappen hat dagegen einen Durchmesser, der etwas gróßer als der normale ist. Die Kapsel ist glatt, ohne irgendwelche Spur von einer Verdickung oder Schrumpfung infolge Cirrhose. Das $\mathrm{Parenchym}$ ist sehr anämisch, mit einer leichten Fettinfiltration und Zentren von Fettnekrose. Auf der Schnittfläche lảßt das Parenchym die kleinen Äste der $V$. porta durch Thrombi eingenommen erkennen.

Die Gallenblase hat verdickte Wande und enthält klare Galle in geringer Menge. Langs des Mesenterium sind alle Mesenterialvenen bis in ihre feinsten Wurzeln durch rote, meist verstopfende, nicht schr adharente Thrombi erfüllt. Dieser breiten venosen thrombosierten Flache entspricht auf dem Dünndarme $50 \mathrm{~cm}$ unterhalb des Duodenum beginnend und gegen den Blinddarm fortschreitend - ein uber I $m$ langes blaurotes Stuck, das ödematöse mit einem fibrinosen Exudat bedeckte Wände hat. Der Inhall dieser Dunndarmstrecke besteht aus schwarzen, mit Blut vermischten Exkrementen. An der Stelle, wo das Mesenterium auf den Dunndarm ubergeht, kann man auf der transversalen Schnittflache die weiten und thrombosierten Venengefaße beobachten. Ton diesem hamorrhagischen Infarkte geht man allmählich zu dem gesunden Gewebe uber. In dem übrigen Teil des Darmes sind die Exkremente flüssig; die Schleimhaut ist anämısch und ctwas odematos. In der $\mathrm{B}$ a u chhohle etwa $4 \mathrm{l}$ zitronengelber Flússigkeit. Die $\mathrm{N}$ i er e $\mathrm{n}$ sind etwas gröBer als normal, aber von normaler Form. Sie können von der Kapsel befreit werden. Ihre Oberfläche ist glatt und die zwei außergewohnlich anamischen Substanzen sind gut entwickelt. Der Uterus ist klein, ,.schleienschwanzförmig“. Schleimhaut und Muskulatur stark anämisch. Eierstöcke und Tuben normal. Harnblase mit anämischer Schleimhaut, enthalt wenig klaren Urin.

Anatomische Diagnose. Mehrfache Thrombosen und Phlebosklerose der $V$. splenica, porta und der Mesenterialrenen, Hämorrhagischer Infarkt des Dunndarmes. Allgemeine Anämie bei der durch Splenektomie infolge Bantischer Krankheit operierten Patientin.

Die Milz ist im Institute für pathologische Anatomie aufbewahrt worden. Aus derselben wurden dann einige Schnitte ent- 
nommen und in Formol, Alkohol, Sublimat und Zucker fixiert. Es wurde noch jenes Stuckchen des Pankreasschwanzes fixiert und eingebettet, welches während der Splenektomie am Hilus adhärent geblieben war und ferner die Gefäße der exstirpierten Milz.

Aus dem Material der Autopsie wurden dagegen Leberschnitte, die intrahepatischen Thrombosen der Porta, die mesenterischen Thrombosen, Pankreas, der mit Infarkt betroffene Darm usw. entnommen.

Es kam zunächst darauf an, die Natur der splenischen Erkrankung genau zu bestimmen. Unsere ersten Untersuchungen wurden daher zunächst auf die Milz und hauptsächlich auf ihr Stutzgewebe gerichtet.

Histologische Untersuchung der Milz. Die splenische Kapsel ist mittelmäßig und gleichartig verdickt. Sie besteht aus ovalen, länglichen, bindegewebigen, längs der größeren Achse des Organs angelagerten Zellen, die beim Austritt etwas zahlreicher werden und mit dem splenischen Gewebe nicht zu verwechseln sind. An der Übergangsstelle zwischen diesem Gewebe und der Kapsel sind kleine Gefäße sichtbar, deren Wände meist nur aus Endothel gebildet ist. Solche Gefäße sind mit Blut gefült und zeigen zwischen den fibrillaren Bündeln der Kapsel abwechselnd Warzenringe. Diese kleinen Warzenkreise sind mit roten und gut erhaltenen Globulis gefillt. Das Bindegewebe der Kapsel färbt sich durch v. Gi es on s Verfahren blaßrot, es zeigt keine Anzeichen von Hyalinentartung. Von der Kapsel strahlen gegen das Innere große bündelförmige Fortsetzungen aus, die das splenische Gewebe senkrecht durchschneiden und außer einer Stutze für die Pulpaelemente noch eine Stutze fur die Milzgefäße bieten. Dieses Bindegewebe nimmt bei den durch v. Giesons Methode gefärbten Präparaten dieselbe Farbe wie die Kapsel an und gleich wie diese ist es sehr arm an Zellelementen.

Indem diese von der Kapsel ausgehenden Bündel tiefer in dic Pulpa hineingehen, nehmen sie nach und nach an Dicke ab. Ihre normale Dicke bleibt nur rings um die Gefảße unverändert, während die Entwicklung der Dicke verhältnismäßig zum Durchmesser der Gefäße bleibt.

In der Pulpa unterscheidet man - wie bekanntlich - die elgentliche Pulpa und die Follikel. Letztere sind, bei der in Untersuchung stehenden Milz zwar sehr spärlich, doch sind sie vorhanden. Sie sind jedoch sehr unregelmäßig verteilt, so daß, während man bei einigen Schnitten gar keine auf dem ganzen mikroskopischen Schnitt wahrnehmen kann, ist es dagegen bei anderen Schnitten moglich auf ein mikroskopisches Feld mehr als einen Follikel zu sehen.

Die Untersuchung der Follikel hatte in unserem Falle ein großes 
Interesse. Wir werden deswegen uber die gemachten Beobachtungen eingehend berichten. In dieser Absicht haben wir zahlreiche Schnitte ausgeführt und der Zweck war klar. Da es sich um eine durch Operation infolge Bantischen Syndroms entfernte Milz handelte, war die Untersuchung des Follikelapparates in erster Linie notwendig, um die Bestätigung der klinischen Diagnose zu bekommen. Inmitten eines vorwiegend fibrösen Gewebes sieht man Follikel mit normaler Struktur, rundlich oder eiförmig, ausschließlich aus Lymphocyten bestehend, die durch ihre Affinitat zu den Farbstoffen sehr deutlich in die Augen springen. Diese Follikel sind untereinander sehr gut begrenzt, fast mochte ich sagen, sie sind ganz vereinzelt. In der Mitte der Follikel sieht man das gut entwickelte Keimzentrum, welches schon unter einer kleinen Vergrößerung sichtbar wird. Dieses Zentrum setzt sich aus IO-I5 Elementen zusammen, die etwas großer als die gewöhnlichen Lymphocyten sind und ein klares Protoplasma, sinen zur globulären Form neigenden Kern und sehr deutliche chromatische Kernkörperchen haben. Ich habe keine sicheren Formen ron Mitosen vorgefunden. Zwischen diesem Keimzentrum der Follikel und den herumgelagerten Lymphocyten ist ein kleines Gefäß vorhanden. Das ist die zentrale Arterie, die sehr klein ist, aber scheinbar normale Wande besitzt. Ihre Tunika, insbesondere die Adventitia, 1st anscheinend gleichmaßig verdickt. Da es sich aber um ein sehr kleines Gefaß handelte. war dieser Befund schwer absolut genau zu bestimmen. Die diese Follikel begleitenden Lymphocyten liegen aufeinander und die sie trennende Intrazellularsubstanz nımmt die Färbung des Bindegewebes nicht an.

An der Peripherie der Follikel ist dagegen die bindegewebige Neubildung deutlich nachweisbar in Form von spindelförmigen oder fibrillaren zu Bundeln vereinigten Elementen, die uberall die Milzpulpa durchschneiden. Wir haben schon erwähnt, daß dieses Bindegewebe in engem Zusammenhang mit den Gefäßen steht. An dem als Norm dienenden Präparat ist gleich unter dem eben beschriebenen Follikel ein Gefảß mit einem mittelmäßigen Durchmesser vorhanden. Dieses Gefäß ist wahrscheinlich nichts anderes als die nächste Strecke der zentralen Arterie bei dem beschriebenen Follikel. Vor der Adventitia dieses Gefäßes sieht man zahlreiche und feinere bindegewebige Streifen, die sich strahlenartig verteilen und auf solche Weise auch in die peripheren Teile der untersuchten Follikel hineindringen. Diese Streifen anastomosieren dann, indem sie Netze bilden, die wenige Pulpaelemente enthalten. Sie sind sehr schmale und zarte Streifen. die durch ihre Verbindungen wahre Zellen fur die Pulpaelemente begrenzen. Sie werden durch v. Gies on s Farbung rot, und weil sie sehr dünn sind und deswegen sofort in die Augen springen, kann man annehmen, daß hier die Farbe sich mit Vorliebe fixiert hat. An anderen Stellen, und zwar viel häufiger, nimmt dieses Netz statt der 
roten Färbung, wie bei dem Fuchsin, eine zwischen roter und gelber Farbe und in seltenen Fällen eine deutliche gelbe Färbung an.

Bei einem zweiten, ebenfalls transversal durchgeschnittenen und von uns untersuchten Follikel haben wir Befunde angetroffen, die verschieden von den bis jetzt beobachteten sind. Es ist nämlich kein Keimzentrum vorhanden und die viel weniger zahlreichen Lymphocyten sind durch eine große und deutliche Menge Intrazellularsubstanz getrennt. Letztere Substanz setzt sich in Verbindung mit der zentralen Arterie des Follikels; sie ist eben nichts anderes als die Fortsetzung der äußeren Tunika derselben. Das Lumen dieser Arterie ist normal, aber ihre äuBeren Schichten sind verdickt und nehmen durch v. Gi esons Verfahren eine rote Färbung an, die sich in Form von schmalen Streifen auch zwischen den Follikelelementen fortsetzen und gegen die Peripherie des Follikels eine rot-gelbe Färbung erhalten.

Wenn wir mit dem Studium des Follikelapparates der in Untersuchung stehenden Milz weitergehen, so bemerken wir immer auf derselben Schnittfläche das Fortschreiten der bindegewebigen Sklerose des Follikel. In dem von mir untersuchten Follikel hat ein solcher Prozeß von Sklerose einen großen Teil desjenigen Gewebes betroffen, der nahe an der Follikelarterie liegt, wo das Bindegewebe außergewöhnlich angehäuft ist. An der Peripherie kommen die miteinander durch schmale und deutliche Streifen verbundenen Lymphocyten wieder zum Vorschein.

Alles das betrifft die Veränderungen in dem Follikelapparat. Wir möchten noch hinzufugen, da $B$ auf vielen mikroskopisch untersuchten Feldern hier und da unregelmäßig zerstreute Follikelreste gefunden wurden, die auf kleine Lymphocytenanhäufungen beschränkt und bald von vorwiegend fibrillärem Bindegewebe durchschnitten, bald von demselben umgeben waren.

In der Pulpa sind die venösen Sinus eng, es fehlt dabei irgendwelche Spur von Stauung, die zwischen den Sinus liegenden Stränge sind deutlich nachweisbar, in Form von Fäden, die manchmal aufeinander liegen, so daß sie in ihrem Aussehen Bündel vortäuschen.

Dic Pulpa wird also durch diese Bündel durchschnitten sein, welche ihr ein fibróses Aussehen erteilen und denselben verdankt sie ihre Festigkeit.

Das Stützgewebe ist in der Milz durch die Kapsel, durch die von der Kapsel zu den Abzweigungen der Milzgefäße entsandten Fortsetzungen und durch die Gefäße dargestellt.

Die mittelstark und gleichmäßig verdickte Kapsel besteht aus unebenen (schwebenden), regelmäßig aufeinander liegenden, bindegewebigen Bundeln. Diese letzteren sind untereinander und mit der Achse der Milz parallel. Sie setzen sich aus Fasern zusammen (durch v. Gi e s on rot gefärbt), die einen platten, ovalen, an chromatischen Körperchen reichen Kern zeigen. 
An dem oberflächlichen Teil der Kapsel sind die glatten Muskelfasern sparlich und noch sparlicher sind die elastischen Fasern. Wo letztere vorhanden sind, sind dieselben ganz kurz und schmal; sie verlaufen in der Richtung der bindegewebigen Bündel der Kapsel. Sie begrenzen diese Bundel durch elastische Netze, die manchmal aus einer einzigen Faser bestehen. Auf solchem Niveau der Kapsel liegen die elastischen Fasern immer vereinzelt und bilden niemals Bundel. Dic kleinen hier und da zerstreuten Gefäße sind auf schmale, von elastischen Elcmenten freie Kapillaren beschräkt, deren Endothel aber gut erhalten ist.

Der tiefste Teil der Kapsel, das ist der dem Milzgewebe angrenzende Teil, unterscheidet sich von dem cben besprochenen dadurch, daß die darin enthaltenen bindegewebigen Bundel nicht nur geschlossener (angehäufter) und reıcher an Zellelementen sind, sondern auch dadurch, daß dieser Teil eine grobere Entwicklung an der Stelle erhält, wo er sich in das Milzgewcbe hineindrängt, um dort die Stutzbalken zu bilden. An der Stelle, wo diese tiefe Kapselzone eine intraparenchymale Fortsetzung bildet, wird dieselbe allmählich bis an die oben crwahnte Stelle dicker, um dann nach und nach eine normale Dicke wieder anzunehmen. Außer den schon erwähnten bindegeweblgen Bundeln tragen zur Vermehrung der Dicke der tiefen Kapselschicht noch die elastischen Elemente und die Gefaße bei.

Die elastischen Fasern nehmen an Zahl und Dicke zu, so daß sie an der Grenzzone des Milzgewebes echte Bündel bilden. Diese umgeben und begleiten die bindegewebigen Bündel und verlaufen in deren Richtung, indem sie sich mit denselben in die Milzpulpa hineinschieben.

Nachdem die in Rede stehenden Bundel in Beruhrung mit den Gefäßen gekommen sind - welch letztere hier an Zahl und Durchmesser sehr vermehrt und nicht nur durch Kapillaren, sondern auch durch kleine Venen und Arterien dargestellt sind - losen sich diese elastischen Bundel in Fibrillen auf. Während diese auf der einen Seite die feineren bindegewebigen Fasern begleiten, ordnen sie sich auf der anderen Seite um die Gefäße ringsherum, indem sie dadurch wahre Häutchen bilden, die die dunnwandigen Gefäße begrenzen.

Ton dem Punkte, wo das Bindegewebe (von dem elastischen begleitet) in das Milzgewebe hineindringt, steht das Verhalten des einen Gewebcs in direktem Verhảltnis zum anderen.

Wo das faserige oder fibrilläre Bindegewebe uberwegend ist, sind auch mittelgroße elastische Bundel überwiegend vorhanden. Wo dagegen die bindegewebigen Fibrillen sich auf das Minimum beschranken, nehmen die elastischen Fasern uberaus an Dicke ab.

Das clastisch-bindegewcbige, in der Milzpulpa liegende Geflecht hauft sich hauptsächlich um die Gefaße und dann um die venösen Sinus an. 
Wir haben schon vorher erwähnt, daß die intravaskulären Stränge gut entwickelt waren. Diese verdanken einer Hyperplasie des fibroselastischen Gewebes ihre Entstehung.

Was die Gefäße anbelangt, so bemerkt man eine ausgesprochene Neubildung von kleinen Gefäßen - Arterien oder kleinen Venen alle von einer angemessenen Umhullung begleitet. Durch v. Gies o n s Verfahren nimmt das die normalen und die neugebildeten Milzgefäße umhüllende Gewebe eine lebhafte rote Farbe an (besonders wenn das Stück in Alkohol oder Formalin fixiert worden ist), während dagegen das Netz der Sinus und der Follikel eine sehr schwache (matte) Farbe erhält.

Gehen wir nun zur Untersuchung der Follikel über, und zwar zur Prüfung ihrer Beziehungen zu den extra-

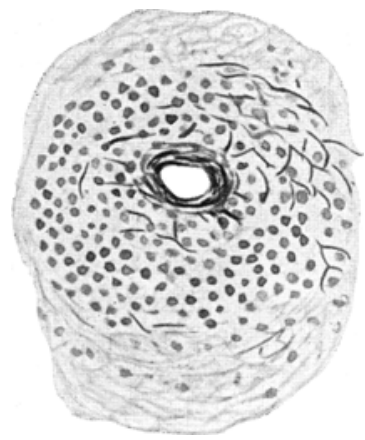

Fig. I.

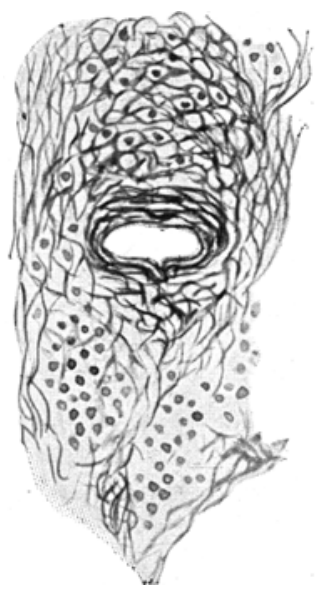

Fig. 2.

Follikelarterien auf verschiedenen Stufen von Fibroadenie.

follikulären Arterien, so treffen wir noch stärkere Befunde von Sklerose, die wir schon oben bei den intrafollikulären Arterien beschrieben haben.

Die Follikelelemente bilden mit der ihnen anliegenden Arterie ein Ganzes, odcr besser gesagt, die außerc, wie gewohnlich verdickte Gefäßwand schickt in die Follikelelemente ihre Fortsetzungen hinein. Diese Wand ist ja teilwcise durch das Bindegewebe adventitialer Entstehung ersetzt, worin wir noch sparliche und unregelmäßig eingeschlossene Lymphocytenelemente unterscheiden können. In dem eben untersuchten Follikel ist die Sklerose sehr vorgeschritten, weil sie in einem an Zellelementen sehr armen, noch nicht gänzlich hyalinen, aber bald hyalin werdenden Gewebe auftritt. Die nach We igert (Fuchsin) und v. Gies on gleichzeitig gefarbten Präparate sind sehr deutlich und klar.

Ich halte mich nun an eine Stelle meines Präparates, wo man 
die Beziehungen der extrafollikulären Arterien zu den Follikeln selbst beobachten konnte:

Zwischen den blaßgelb gefärbten Follikelelementen kann man zahlreiche und schmale ziegelrote (das ist die vom Gewebe angenommene Farbe, wenn es mit der Weigertschen Flussigkeit behandelt wird) Linien beobachten, welche sich zwischen die Follikelelemente schlangenartig winden, durch die Hauptmasse der Follikel selbst eindringen und an einigen Stellen auch bis zur entgegengesetzten Peripherie gelangen. Diese Linien verlaufen bald vereinzelt und bald

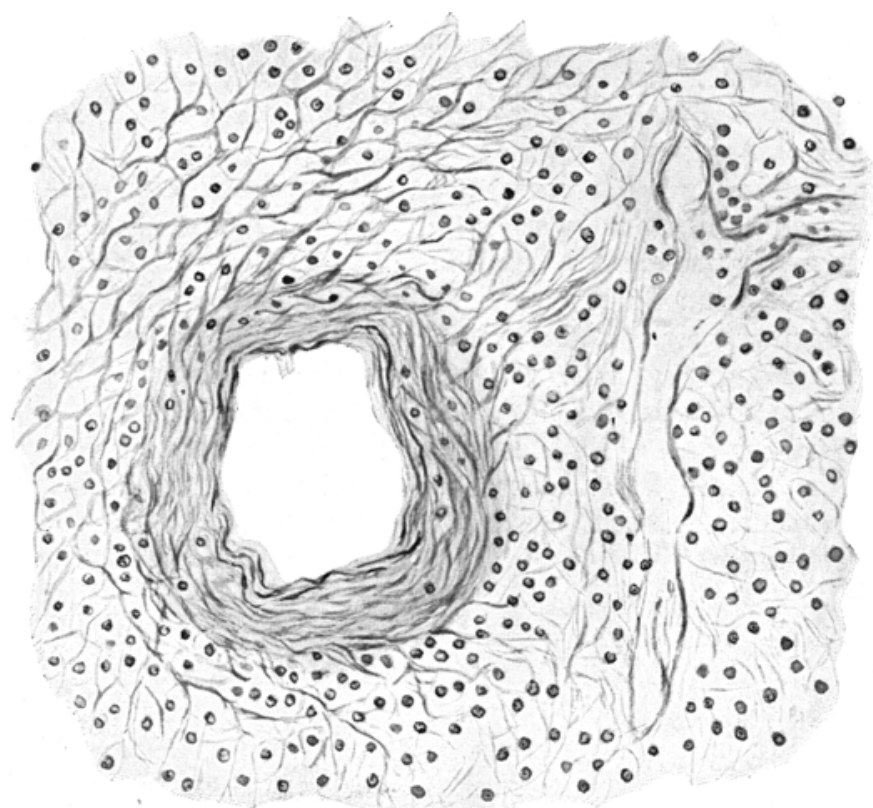

Fig. 3. Follikelartenen auf verschedenen Stufen von Fibroadenie.

anastomosiert. Im letzteren Falle umkreisen sie eine Gruppe, die nicht mehr als 6-8 Lymphocyten enthält.

Die Farbung dieser Fibrillen ist nicht gleichmäßig, sondern auf einigen Strecken ziegelrot, auf anderen dagegen rot-gelb. Das ist an der außeren Pcripherie des Follikel, und zwar besonders auf der entgegengesetzten Seite der Arterie der Fall, wo die Färbung eine deutlichere wird, weil die Zahl der Fibrillen viel geringer ist. Die Fibrillen ziehen wellenformig oder schlangenartig. Ihre Dicke ist immer eine sehr geringe, dieselbe neigt zur Abnahme.

Wenn wir dagegen von dem Follikel aus nach der Arterie hin schauen, so sehen wir, daß die Scheidewände (Septen) verdickt 
werden und eine dauernde dunkelrote Farbe annehmen. Die durch dieselbe begrenzten Warzenringe werden infolge der fortschreitenden Verdickung der Septen immer kleiner, so daß die Anzahl der eingeschlossenen Lymphocyten sehr beschränkt wird. Manchmal können wir auch ganz einwandfreie Septen oder Warzenringe sehen.

An dieser Stelle verfilzt sich dieses Bindegewebe (oder netzartiges Gewebe) mit der Adventitia der extrafollikulären Arterie.

Aus dieser Beschreibung erhellt deutlich, daB von der Arterie, und zwar von deren äußerster Tunika, ein netzartiges Bindegewebe seinen Ursprung nimmt und in den nächsten Follikel eintritt. Zunächst besteht dieses Bindegewebe aus groben und gut gefärbten und dann aus immer schmaler werdenden Maschen, die zuletzt an der Peripherie der Follikel selbst sehr klein und schwach werden.

Dieses Netzgewebe schließt die Lymphocytenelemente zunächst in weite und dinne Maschen ein, welche dann, sich der Arterie nahernd, immer dichter werden, aber doch stets, auch dicht an der Arterie, Lymphocyten enthalten. Die Folge davon ist einleuchtend und klar: Sklerose des Follikels selbst. Dieser Prozeß hat seinen Ursprung an der follikulären Arterie oder zwischen den Follikelelementen. Es genügt, die von mir gegebene Beschreibung zu verfolgen, um zu ersehen, da $B$ die vorgeschrittenen Veränderungen auf der äußeren Seite des Gefäßes nachweisbar waren und daß sie sich von diesem Gefäße fast fächerartig zwischen den follikulären Lymphocyten verbreiten. Auf dieser Stelle mußte also die Veränderung ihre Entstehung gehabt und von dort aus sich dann weiter verbreitet haben.

Wir bemerken noch, daß es uns unter normalen Verhältnissen durch die gewöhnliche Färbungsmethode (v. Gieson) niemals gelungen ist, ein einziges Netz deutlich zu beweisen. Der Beweis eines solchen ist nur durch die für das Bindegewebe speziellen Methoden möglich. Bei meinem Falle konnten die Netze sogar durch die gewöhnlichen Färbungsverfahren zur Ansicht gebracht werden.

Damit sich dieses Netz unter solchen Umstanden deutlich macht, muß es verdickt sein - indem es seine chemische Beschaffenheit behall - was ich glaube, oder es muß seine Eigenschaft ändern und sich dem kollagenen Bindegewebe nähern. Wahrscheinlich kann auch diese zweite Annahme richtig sein. Sie widerspricht nicht der anderen Annahme, wenn wir nur daran denken, daß das Netzgewebe und kollagene Gewebe - wenn auch chronisch verschieden - zu derselben Familie gehören und daß eine Metaplasie des einen in das andere Gewebe a priori nicht ausgeschlossen werden kann.

Die histologische Methode, welche die zartesten bindegewebigen Fibrillen zur Ansicht bringt, ist die Bielschow skysche Methode. Ich habe sie zur Untersuchung dieser Milz angewendet. Darüber werde ich aber weiter unten berichten. 
Bevor ich zum Studium des Stutzgewebes schreite, ist folgendes als bedeutungswert zu erwähnen: Die fibros-adenoide Umgestaltung des Netzes beginnt in den follikulären Arterien und von dort erstreckt sie sich auf die Follikel und erst dann auf die Pulpa. Einen zweiten Beweis dafur haben wir in der unten angefuhrten histologischen Beobachtung.

Wir können namlich verschiedene mikroskopische Serienschnitte durchsuchen, ohne daß wir einen fibrös-adenoiden Befund in der Pulpa oder in den Follikeln nachweisen, und dann sehen wir plötzlich auf einem fast gänzlich aus Pulpaelementen oder intravaskulären Strangen zusammengesetzten Felde eine vereinzelte follikuläre Arterie hervortreten, von deren Peripheric die fibrös-adenoide Umänderung der Follikel und der Pulpa außerhalb des Mittelpunktes beginnt. Bei den zu einem follikularen System nicht gehörcnden Arterien bemerkt man nic an der Peripherie des großen, durch die Adventitia dargestellten Muffes eine fibrose Adenie der herumliegenden Pulpa. Die zu enem follikulären System nicht gehörenden Arterien unterscheiden sich von dem zu einem solchen gchorenden nicht nur dadurch, daß erstere einen verschiedenen Durchmesser haben, sondern auch dadurch, daB sie immer von Venen begleitet sind, und weil keine Follikel nachzuweisen sind, wic es bei den traubenartigen Arterien der Fall ist. Wenn es manchmal moglıch ist, eine fibröse Adenie der Pulpa nachzuweisen, so steht sie in Beziehung mit follikulären Arterien, die zu einigen naheliegenden, von diescm Prozeß betroffenen Feldern gehören.

Fur die Untersuchung des dunnen Stutzgewebes habe ich das M a llor y sche Hamatoxylin, die M a ll or y sche Methode mit saurem anilinblauen und orangen Fuchsin angewendet, wobei ich aber, statt des sauren Fuchsins, dic Unnasche Polychromie mit neutralem Orcein und die Bielschows ky sche Behandlung benutzt habe.

Was die letzte Methode anlangt, habe ich die von $\mathrm{Levi}$ vorgebrachte Änderung befolgt, indem ich, wie B arbacci, alle verschiedenen Momente des Prozesses auf dic in Paraffin eingebetteten Schnitte untersuchte. Ich habe ferner die alte MIareschsche Untersuchungsmethode angewendet, die mir aber keinen konstanten Erfolg gab, vielleicht deswegen, weil die in einem Berichte zusammengestellten Einzelheiten der Mareschschen Theorie nicht genau angegeben waren. Alle oben angeführten Methoden geben gute und gegenüber der v. Gi es on s bessere Ergebnisse, die aber nicht konstant sind und infolge ron ganz geringen und deshalb leicht zu übersehenden Gründen ron Präparat zu Präparat wechseln können. Die Bielschowskysche Methode hat mir dagegen immer gleichmäßige Erfolge gegcben. Die v. Gieson-Methode dient vorzüglich zum Nachweis fur das gewöhnliche Bindegewebe, aber mit den anderen Methoden verglichen, ist dieselbe ungenügend, um die zarten Fibrillen 
zu beweisen. Wenn wir die v. Gies on-Färbung mit der Weigertschen kombinieren, so erleichtert das den Nachweis des dünnen Bindegewebes. Das Weigertsche Hämatoxylin und das darauf folgende v. Giesons erleichtert überaus die Färbung des Bindegewebes dadurch, daß sich die verschiedenen Farbstoffe mit der außersten Deutlichkeit differenzieren. Aber die mit dem Malloryschen Hämatoxylin und noch mehr mit der Bielschowskyschen Methode erreichten Ergebnisse sind bei weitem besser. Diese Methode hat die allen Silberpräparaten gemeinschaftlichen Fehler, und die Schwierigkeit der Färbung der Zellelemente und infolgedessen können die genauen Verhältnisse zwischen Zellelementen und bindegewebigen $\mathbf{F a}$ sern schwer bestimmt werden.

Wenn wir aber Methoden fuir Vergleichspräparate anwenden, kann diese Mangelhaftigkeit beseitigt werden.

Wir haben nun die Verdickung des Milzgewebes bei den Follikeln wie auch bei der Pulpa beschrieben. Es erübrigt uns noch die Untersuchung dieses Netzes durch angepaßte histologische Methoden und insbesondere durch die Bielschowskysche Methode zu erörtern. Letztere stellt vorzüglich die Feinheit des Netzes, jene sehr kleinen fibrillären Fasern dar, die heutzutage unter dem Namen „Gitterfasern" trefflich bezeichnet werden.

Gitterfasern nennen (im engsten Sinne des Wortes) die Patho. logen jene Fasern, die, im allgemeinen gesprochen, als Stütze der parenchymalen Elemente dienen. Dieser Name ist in allgemeinen Gebrauch gekommen, nachdem spezielle histologische Mcthoden uns diese Fasern mit einer gewissen Leichtigkeit, sei es unter normalen, sei es unter pathologischen Verhältnissen, klar gezeigt haben. Das Bestehen dieser Fasern war aber schon vorher den alten Histologen bekannt. die durch heute fast aufgegebene Methoden (Verdauung, Aufweichen, Auspinseln) dieselben beim tierischen Organismus schon erblickt und beschrieben haben.

Das war der allererste Begriff, den man sich von den Gitterfasern gemacht hatte. In den letzten Jahren hat sich dieser Begriff nach und nach erweitert und man hat ihn - immer mit der Bedeutung eines Stützelementes - auf jene bisher für hyalin gehaltenen Membranen, welche die Tubuli einiger Drüsen umgeben, und ebenso auf die Basalmembranen der kapillaren Endothele ausgedehnt.

Es war also natürlich, daB nach der Anwendung der Bielschowskyschen Methode für die Neurofibrillen und nach den von M a resch in Deutschland und Levi in Italien herbeigeführten Än. derungen, um diese Gitterfasern auch bei menschlichen Geweben zu beweisen, jene Organe oder Parenchyme zunächst und genau studiert wurden, worin unsere alten Histologen durch die oben erwähnten Methoden das Vorhandensein dieser speziellen Fasern schon erblickt oder bewiesen hatten. Die gewöhnlichen histologischen Methoden 
waren nicht imstande, diese Fasern zur Klarheit zu bringen, und auf solche Weise sind die Leber und die Milz (viel mehr die erste als die zweite), sei es auf normalem oder pathologischem Wege, seitens der Pathologen der Gegenstand dieser Vergleichsuntersuchungen geworden.

Unter den Anatomen hat nur Wolff (nach Russakoff) uns cine kurze Darlegung uber die fibrilläre Struktur der Leber des Frosches gegeben. Bei den Untersuchungen haben sich naturlich hauptsachlich die Pathologen betatigt, weil es nur aus den Studien uber das verschiedene Verhalten der Gitterfasern bei krankhaften Prozessen moglich war, die verschiedenen Einzelheiten klarzulegen, um dann móglicherweise zu deren Deutung und Erklarung zu gelangen.

Zum besseren Verstandnis der Bedeutung dieser Gitterfasern als Stützelement der parenchymalen Elemente werden wir uns auf das eingehender untersuchte Organ, die Leber, beziehen, welche Gegenstand größerer Streitigkeiten gewesen ist.

Das Netzsystem der Gitterfasern steht in der Leber in Beziehung zu den zellularen Trabekeln (wobei es fur die parenchymalen Elemente des Organs eine Stutze darstellt) und mit interlobulären Kapillaren, indem es dieselbe umgibt und um sie herum jenes Netz bildet, das von den Deutschen ,Adventitia capillaris" genannt wird. Kupfer (s. Russakoff), der als erster einen den parenchymalen Leberelementen als Stutze dienenden Einschlag beschrieben hat, unterscheidet zwci Arten von interlobulären Fasern:

I. Die umspinnenden Fasern, welche die interlobulären Kapillaren umwickeln und dieselben miteinander vereinigen.

2. Die radiären Fascrn, die, nach $\mathrm{K}$ u p fer, einen Verlauf hätten, welcher unabhangig ron dem der Kapillaren ware und sich zwischen die hepatischen Zellen hineinschiebt.

Die Meinungen uber die gegenseitigen Beziehungen der beiden Fasersysteme, uber deren Abgrenzungs- und Anastomosenart und über ihre Beziehungen zu den Lymphokapillaren sind sehr abweichend. Wahrend die einen, und zwar die jungsten, die Kupfersche Deutung befolgend ( $M$ a resch, Herxheimer, $\mathrm{Sch}$ id $\mathrm{t}$ ), nur nach den die Blutkapillaren umspinnenden Gitterfasern forschen (M a reschs sogenannte Adventitia capillaris), ziehen die anderen noch die Gitterfasern in Rucksicht, welche uberwiegend in Zusammenhang mit den Trabekcln der hepatischen Zellen stehen. Nur wenige haben die möglichen Beziehungen der Gitterfasern zu den Wänden der perikapilläen Lymphraume studiert. B a r b a c ci selbst, der durch die Methode der Silberdurchtränkung die harte, arteriosklerotische Leber untersucht hat, nimmt die Unterscheidung zwischen umspinnend e $n$ und radial e n Bundeln an, wobei er als' ,r a d i a l" die dickeren und starken Bündel bezeichnet, die sich vom Zentrum des Läppchens nach deren Peripherie richten, um sich hier in die kollagenen Fasern 
des interlobulären Bindegewebes fortzusetzen. „Umspinnend“ heißt er die dünneren und zartesten Bundel, welche, von den ersteren ausgehend, nach seiner Meinung die interlobulären Kapillaren und die hepatischen Trabekeln umwickeln, jedoch mit dem Unterschied, $\mathrm{da} B$ die balkenartigen dichter und die um den Kapillaren befindlichen spärlicher und aus einzelnen Bündeln zusammengesetzt sind.

Unter dem pathologischen Gesichtspunkte, so schreibt Barbacci, scheint jedoch diese Unterscheidung mehr eine übersichtliche als eine reelle zu sein.

Wir haben nun erklärt, was man unter dem Namen ,Gitterfasern" verstehen soll, und die verschiedenen Beziehungen derselben zu den parenchymalen Elementen eines Organs und zu den entsprechenden Kapillaren erörtert.

Aus den von uns darüber konsultierten Verfassern wollen wir im folgenden das Verhalten dieses Stützgewebes bei den Organen erwähnen (insbesondere bei der Leber, der Lunge und den Lymphdrüsen), die zu diesem Zweck untersucht worden sind.

In der Leber (Russak off) zeigen sich die Gitterfasern aus allerfeinsten Fibrillen zusammengesetzt, die auf der einen Seite die hepatischen Trabekel, auf der anderen Seite die Kapillaren bedecken. Dieselben sind nicht frei, sondern miteinander durch eine hyaline Substanz verbunden. Das Vorhandensein und die Bedeutung dieser Substanz wird verschieden gedeutet. Darauf werden wir später näher eingehen.

An der Peripherie des Lappens und um die zentrale Vene erleiden diese Fibrillen eine leichte Verdickung. Der Umstand, daß die Gitterfasern sich im Umkreis der äußeren Fläche der hepatischen Trabekel befinden, hat die Annahme als wahrscheinlich erscheinen lassen, da $B$ diese Gitterfasern mit jenen hyalinen und eigenen Membranen verglichen werden können, die das periphere Ineinanderweben zur Stützung einiger Drüsen, z. B. der Speicheldrüsen und der Niere bilden. Eine solche von einigen bestätigte und von anderen als eine künstlich angesehene Annahme wird auch von $R$ us sak of $f$ verfochten und ferner von $\mathrm{Maresch}$ bestätigt. Letzterer Autor soll ja bei giftigen Ödemen und bei Stauungsödemen eigentümliche Safträume zwischen den hepatischen Zellen und den perikapillären Fasern bemerkt haben. Diese Räume haben keine zelluläre Bekleidung und nichts mit den Lymphspalten zu tun. Er deutet dieselben als infolge einer Trennung des Epithels von der eigenen Membran gebildete Räume. Diesen Befund können wir infolge Veränderungen an der Leiche beobachten.

Bei den hepatischen Veränderungen wurde hauptsächlich das Verhalten der Gitterfasern studiert und von diesem pathologischen Gesichtspunkte aus sind es natürlich diejenigen gewesen, die in letzter Linie zu einer Zunahme der Stützsubstanz im allgemeinen fuhren mußten. 
Unter diesen verschiedenartigen Prozessen wurden dann diejenigen gewahlt, bei dencn dic Silberdurchtränkung die eigentum. liche Verhaltungsweise der Gitterfasern zeigte. Durch dieses Verhalten waren im Grunde genommen jene Erscheinungen zu erklären, die sich durch die gewohnlichen histologrschen Methoden nur unbefriedigend erklären ließen. Ich erwähne dabei die harte, arteriosklerotische Leber, welche in Italien von B a b a c ci untersucht worden ist.

Diesem Verfasser waren die anatomischen Eigentümlichkeiten dieser Leber schon bekannt. Er hatte sich aber immer uber den Umstand gewundert, daß man durch die gewohnlichen histologischen Methoden niemals , in dem eigenen Bindegewche des Organs den letzten und innigsten Grund" fur die Hàrte gefunden hat, welche die makroskopische Eugentimlichkeit des Bindegewcbes ist. - Er hat dann die obengrenannten Veranderungen durch die BielschowskyLevische Methode untersucht, wobei er cine von ihm greänderte Methode angrewendet hat, welche den Unterschied zwischen den Gitterfasern und kollagenen Fasern mit größter Leichtigkeit und Genauigkeit ermoglichte. Er hat dabei einc ausgr'sprochene Proliferation des Netzgewebes der in Untersuchung stehenden Organe nachweisen konnen. Diese Proliferation war durch Hypertrophic- und Hyperplasiecrscheinungen gekennzeichnet. Dieselbe zeigte sich aber sprungweise, d. h. sie war nicht gleichmaßig auf dic ganze Flache des Organs verteilt, sondern auf einer Stelle mehr uberwiegend als auf einer anderen und umgekehrt; bald traf dieselbe das Zentrum des Läppchens, bald die Peripherie desselben.

B a r bacei bemerkt ferner, daß durch diesen Prozeß die umspinnenden, wic auch die radialen Bundel getroffen werden. Während aber bes den ersteren die Hyperplasicerscheinungen vorwiegen, finden wit bei letzteren die Hypertropheerscheinungen uberwiegend.

Dur zuerst von Russle bemerkte und später von anderen Verfassern (unter denen auch Barbacci) bestatigte Umstand, daB einige Bundel sich nach Bielschowsky, wie auch (obgleich sehr schwach! nach v. Giieson färbten, bewog Barbacci zur Annahme, daß die von ihm bei der arteriosklerotischen, nach der Bielschowskyschen Methode behandelten Leber, bemerkten Veranderungen der Gitterfasern, sich mit der Zeit in eine echte und eigene Cirrhose umwandeln konnen, weil er manchmal ,,bei durch v. Gieson stark gefärbten Praparaten das mehr odcr weniger rosig gefärbte Netz der Gitterfasern beobachtet hat".

Der Umstand, daß einige Bundel sich durch beide Methoden färben lassen, ist von $\mathrm{R} \ddot{\mathrm{s} s \mathrm{l}} \mathrm{e}$ als eine Neigung der Metaplasie der Gitterfasern zu kollagenen Fasern gedeutet worden.

Die Befunde, worauf B a r ba c ci eine solche Möglichkeit gründet, haben einen gewissen Wert, weil er in solchen Fällen die absolute Abwesenheit irgendwelchen Hindernisses am portalen Blutumlauf und 
das Vorhandensein einer hochgradigen Arteriosklerose bemerkt hat, welch letztere sich auch bis auf den Hauptzweig der hepatischen Arterie ausgedehnt hatte. „Zur Erklärung der Seltenheit der parenchymalen Veränderungen in der Leber arteriosklerotischer Natur obgleich die Leberarterien, insbesondere die mit einem mittelmäßigen Durchmesser, so häufig von einem ArterioskleroseprozeB getroffen werden, wie es bei anderen Organen der Fall ist - haben wir hier die Erscheinung des doppelseitigen, in diesem Organe vorhandenen Blutumlaufes, da in theoretischer Hinsicht nichts gegen die Behauptung spricht, daß ein eigener Cirrhoseprozeß - wie es bei anderen Organen der Fall ist - sich in der Leber auf Grund nur arteriosklerotischer Veränderungen abwickeln könne (B a r b a c ci)."

Es wäre natürlich nicht zwecklos, wenn wir diese Untersuchung auch auf andere gewöhnliche Formen von Cirrhose, z. B. auf die renalen, ausdehnen, um zu sehen, ob bei denselben eine Proliferation der Gitterfasern zum Prozeß der Cirrhose Veranlassung gibt.

Bei verschieden hepatischen Hypertrophien hat Rössle die Verhaltungsart der Gitterfasern untersucht. Insbesondere bei jener Hypertrophie, die er als eine wahre Hypertrophie der Leber bezeichnet (mit der hydropathen Herzhypertrophie oder mit der hypertrophischen Leber der schwangeren Weiber oder der Wöchnerinnen, aber nur Begriffsähnlichkeit halber, zu vergleichen) hat $\mathrm{R}$ ös sle eine ausgedehnte Verdickung der Gitterfasern, sei es bei den umspinnenden, wie auch bei den radialen Bundeln beobachtet. Die Gitterfasern färbten sich dann gleichzeitig durch die v. Gi e s o n - Methode (Hyperfärbung).

Dadurch erhielt man ein Kollagenwerden der Gitterfasern und besonders der der Adventitia capillaris angehörenden Gitterfasern, das heißt, man erhielt eine Strukturanpassung, wozu (nach Rössle) ein funktioneller Anreiz Veranlassung gegeben hatte.

Die über die Gitterfasern der Lung e und noch mehr über die Lymphdr üsen gemachten Untersuchungen sind für unser Studium noch wichtiger. Die Streitigkeiten von der Struktur der die Höhle begrenzenden Membran sind schon bekannt; wenn wir uns aber auf die Orzosschen Untersuchungen stutzen - die die vollständigeren sind - so meint man heutzutage, da $B$ diese Struktur teils aus kollagenen, teils aus elastischen Fasern besteht. Durch die Bielschowskysche Methode offenbart sich unter dem Epithel ein feinmaschiges Netz, welches aus sehr feinen, miteinander anastomosierenden Fibrillen zusammengesetzt und auf dem seltenen Bindegewebe gelegen ist (in der Kinder- und Fötallunge reichlich vorhanden), das zwei Alveolen voneinander trennt. Ein solches Gitterfasernsystem ist bei den verschiedensten pathologischen Prozessen der Lunge untersucht worden ( $\mathrm{R}$ us a k off), wie z. B. bei akuten Prozessen infolge Stauung, Emphysem, Tuberkulose und Carcinom. 
Diese Untersuchungen haben die chemische Natur jener Prozesse und deren Unterschied vom kollagenen Bindegewebe bestätigt. Die infolge mechanischer Ursachen auf die Gitterfasern ausgeubten Veränderungen bestehen aus einer Verdünnung in der Hyperspannung der Alveolen (Luftgeschwulst) und aus einer Verdickung und Vernichtung in allerlei Atelektase. Die Festigkeitszunahme, die wir bei der braunen Verhärtung bemerken, gründet sich wenigstens teilweise auf eine Zunahme und eine Verdickung des Ineinanderwebens der Gitterfasern.

Bei der Lungentuberkulose und dem Lungenkrebs haben wir eine starke Neubildung der Gitterfasern.

Auf Grund von Russak offs, Rössles und Joschidas Untersuchungen verlaufen die Gitterfasern in die Gewebe nicht ganz vereinzelt, sondern sie waren von einer halbflüssigen Substanz begleitet oder umwickelt, welch letztere sich durch die Bielschowskysche Methode grau gefärbt. Diese Substanz wäre bei dem ruhenden Netz spärlich und besser sichtbar auf den Stellen, wo wir die Faserneubuldung haben. Diese halbflussige Substanz würde in den Lymphdrusen und in der Milz in Beziehung, ja in dırekte Berührung mit länglichen Zellelementen kommen, deren Protoplasma sich durch die Bielschowskysche Methode grau färbt (während das Kollagen sich braun färbt) und deswegen mußte man die Substanz als eine Fortsetzung des zellularen Protoplasmas ansehen.

Die Gitterfasern sollten also von diesen ,,Mutterzellen der Gitterfasern" abstammen, auf deren Fläche dieselben sich bilden und mit denen sie - bei der späteren Entwicklung - durch die oben beschriebene Substanz verbunden bleiben. Im Verlauf der Entwicklung verschwinden die meisten Zellen und es bleibt nur die fibrilläre Trabekelanordnung dauernd bestehen.

„Diese Bildungszellen konnen weder von den adventitialen Kapillar- und Gefaßßellen noch von den Endothelien der Lymphbuchtungen differenziert werden; dieselben werden als gleichgültig betrachtet."

Nur wenn die Gitterfaser sich von der eigenen Bildungszelle befreit hat, kann sie die Silbertränkung in sich aufnehmen.

Rössle und Joschida haben I40 Drüsen von 66 verschiedenen Fällen untersucht, wobei sie das Vorhandensein der Lymphoidensubstanz in den Buchtungen und an den kapillaren Wänden eines durch v. Gieson deutlich gezeigten Netzes bestätigt haben.

Dieses Netz ist auf dem entsprechenden Teil der Kapsel spärlich und wird auf der Höhe der Lymphbuchtungen und der Follikel reichlicher, während die Keimzentren sehr wenige Fasern enthalten. Die Lymphdrüsen nehmen daran durch Veränderungen der Gitterfasern auf àhnliche Weise teil, wie wir es in der Lunge gesehen 
haben. Im Falle einer Atrophie oder Involution werden dieselben größer, bei Ödem oder Anschwellung dünner und bei übermäßiger Spannung der Lymphbuchtungen können sie sogar zerbrechen. Im allgemeinen sind diese Lymphdrusen sehr widerstandsfahig, so daß wir sie auch bei einer ausgedehnten Zerstorung der Lymphoidzellen ganz erhalten finden können. Ebenso bestehen sie auch bei einem käsigen Gewebe. Bei Prozessen von drüsiger Hyperplasie (Leukämie usw.) haben Rössle und Joschida keine Veränderungen des Netzes der Gitterfasern bemerkt. Deutlich ist dagegen die Proliferation der Gitterfasern bei der Drusentuberkulose, bei den akuten oder chronischen Lymphadenien, bei der Altersatrophie und viel mehr bei der syphylitischen Lymphadenie, wo wir in den Fasern der Lymphbuchtungen, in den radialen, von der Kapsel ausgehenden Fasern, in den Hilusfasern und in den Fasern des eigentlich genannten Lymphoidgewebes (welche die dunncren sind) gleichzeitige Hypertrophie- und Hyperplasieerscheinungen haben.

Rössle und Joschida betrachten daher die Gitterfasern als ein vorläufiges Stadium des kollagenen Gewebes, in welches sich dieselben infolge Metaplasie umwandeln können, wie wir bei den Entzündungsprozessen der Drüsen häufig bemerken können.

Die Zellen, von denen die Gitterfasern abstammen, können, wie schon hervorgehoben, von den Endothelien der Lymphräume nicht unterschieden werden; im Proliferationsfalle sind die Gitterfasern den Epithelzellen (bei der Tuberkulose) und den Fibroblasten gleich.

Ich habe die Bielschowskysche Methode auf die Milz angewendet, die Gegenstand dieser Untersuchung war. Mehr als eine eingehende Beschreibung der mikroskopischen durch diese Methode vorbereiteten Präparate geben uns eine klare Vorstellung die Práparate selbst, welche in der Ärztlichen Gesellschaft zu Parma (bei der Sitzung vom April I9I I) vorgezeigt wurden. Das ganze Netz der Gitterfasern ist, sei es auf der Höhe der Buchtungen oder Follikel und der Kapillaren, verdickt.

Wir konnen auch hier die Unterscheidung zwischen umspinnenden und radialen Bündeln wiederholen, aber dieselbe zeigt sich mehr als je als eine theoretische. Auf dem Niveau der Buchtungen und der Blutkapillaren ist diese Verdickung deutlich nachweisbar und offenbart sich durch Hypertrophie- und Hyperplasieerscheinungen.

Die Fasern sind äußerst schmal und zu Bündeln vereinigt und haben einen mittelgroßen Durchmesser. Manchmal ist eine Buchtung durch eine einzige Faser, aber häufiger durch zwei oder drei aufeinanderliegende und von hellen Räumen getrennte Fasern umschrieben.

An der Peripherie der Buchtung zerfallen diese Fasern in eine Gruppe allerfeinster Fibrillen, welche die Kapillarräume umkreisen, wobei die oft brückenförmig von einer Kapillare zur anderen übergehen. 
Das perikapillare Geflecht hat keine bestimmte Richtung, aber die weit anastomosierten Fibrillen zweigen sich ab und schlagen sich, überall exzentrisch sich zerlegend, auf die nächste Buchtung. Dieselben besitzen offenbar Beziehungen mit den kapillaren Endothelien, wie auch mit den Buchtungen. Sic grenzen unmittelbar daran und so erklärt sich die Bildungsstatte der Gitterfasern. Was die oben angefïhrte Beziehung zwischen Bildungselementen und Zellprotoplasma betrifft, werde ich unten berichten. Auf dem Niveau der Follikel zeigt das Netz gleiche Charaktere, es ist aber nicht so fein und begrenzt wie auf dem Kapillarniveau, sondern es ist verdickt und verdichtet. Die lymphoiden Elemente sind gut zu erkennen und ein jedes ist vom Netzsystem fast gänzlich umhüllt.

Manchmal sieht das Nctzsystem hier und da zerstückelt aus, was vielleicht als eine pathologische Erscheinung anzusehen ist.

Die Bielschowskysche Methode braucht keinen weiteren Beweis fur diese feinen Fibrillen, da dieselben an den Vergleichspräparaten nach v. Gieson und $\mathrm{M}$ allory erkennbar sind. Durch diese zwei Färbungsmethoden treten nur die dickeren Fasern, und zwar jene der Buchtungen deutlich hervor (manchmal aber auch eine kleine Anzahl der dunneren Fasern, wenn wir der v. Giesonschen Farbung dic Weigertsche Fuchsinfärbung vorausgehen lassen), wahrend die ein sehr feines Geflecht bildenden Kapillarfasern auf keine Weise aufgedeckt werden. Es ist ja möglich, auf dem Niveau der perikapillaren Fibrillen jene grau gefärbte Substanz wahrzunehmen, die für die deutschen Verfasser cin Sekretprodukt des Protoplasmas der die Gitterfasern bildenden Zellen wäre.

Was nun den zweiten Umstand anbelangt, d. h. die Metaplasieerscheinungen bei einem kollagenen Gewebe, daruber kann ich leider nichts Genaucs mitteilen. Die Metaplasieerscheinungen sind durch eine ins Braune fallende Farbe der Fasern ausgezeichnet. Es ist aber sicher, daß die dicken bindegewebigen $B$ undel (die sich von der Kapsel in das Parenchym erstrecken) und cbenso auch das die äußere Gefaßtunika bildende Bindegewebe durch die Bielschowskysche Methode einen Farbenton annimmt, der ganz verschieden von der schwarzen Farbe ist, die die Gitterfasern anzunehmen pflegen. Dieser Farbenton ist braun-veilchenblau.

Wenn aber diese Bemerkung bei den Bündeln mit mittelmäBigem Durchmesser möglıch ist, wird die Sache eine uberaus schwierige und zweifelhafte, wenn wir eine kollagene Fibrille von einer Gitterfaser (welch letztere nichts anderes ist als eine vorkollagene Fibrille) auf Grund eines Durchtränkungsprozesses unterscheiden müssen, welcher, obgleich er einen beständigen Farbenton erzeugt, keine absolut bestimmte braune oder schwarze Farbe auf allen Stellen des Präparats erreichen kann.

Durch v. Giesons Hyperfärbung oder Ätzung durch Weigert- 
sches Fuchsin ist es uns gelungen, die Fibrillen eines Malpighischen Follikels rot oder rosig zu färben. Diese Fibrillen zeigten schon Erscheinungen von Kollagenwerden, nämlich von Metaplasie, und außer der Farbenverschiedenheit, die wir bei dem durch die Bielschowskysche Methode behandelten Präparate haben, war es uns auch möglich, diese Metamorphose durch Vergleichspräparate zu verfolgen (Fig. 4).

Bei der von uns untersuchten Milz ist die Proliferation des Netzgewebes nur bei der Durchtränkungsmethode sehr reichlich und deut-

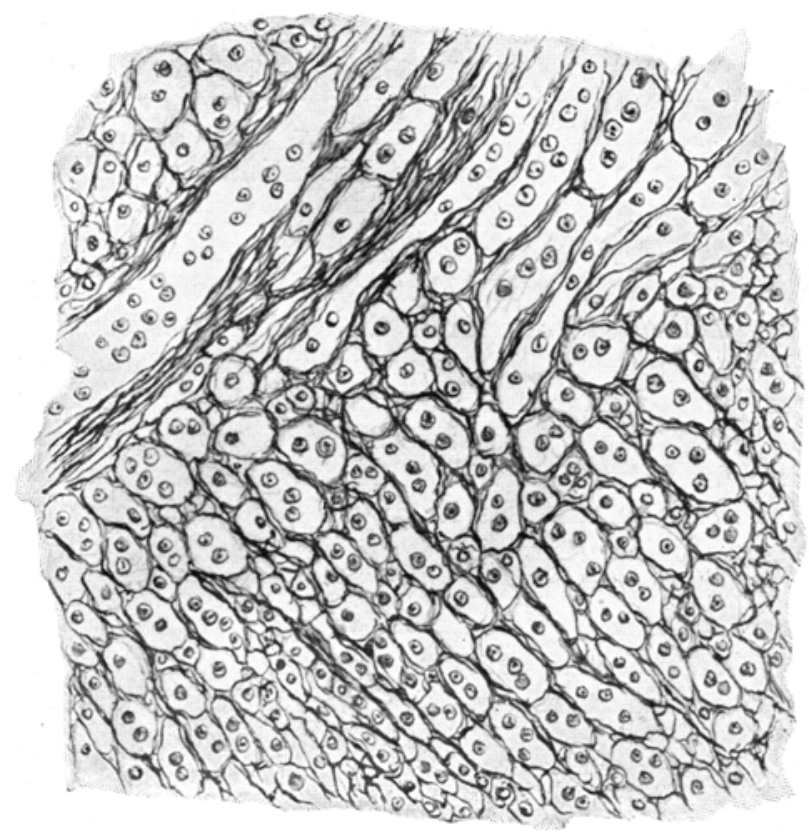

Fıg. 4. Milznetz nach Bielschowsky gefärbt.

lich und es erscheint der Vergleich ( $R$ ussak of $f$ ) mit einem zellular-fibrösen S y n c y ti u m gerechtfertigt, dessen Zellelemente nur bei Regenerationserscheinungen auftreten, während unter normalen Verhältnissen die fibrinösen Elemente mit dem Alter an Masse zunehmen. Die Ähnlichkeit des Netzgewebes mit dem Kollagen ist verschieden groß und das erklärt die Möglichkeit der obenerwähnten Metaplasie. Es sind uns aber auch die von Mall über die Leber, die Niere und die Milz gemachten Untersuchungen bekannt, welche den großeren Widerstand des Netzgewebes gegen die Säuren, im Vergleich mit dem Kollagen, das Fehlen von Leim durch Sieden bestätigt haben. 
Durch Sieden wird ferner dieses Gewebe nicht so rasch wie das Kollagen zerstort.

Ich werde jetzt die Hyalinentartung besprechen. Die oben erwähnten deutschen Autoren deuteten diese auf Grund der Befunde, die sie durch Anwendung der Bielschowskyschen Methode bei den Lymphdrusen erreichten, als ein Sekretprodukt der Bildungszellen der Gitterfasern. Dieses Produkt umgibt zunachst die Bildungszellen ganz ahnlich einer granulosen grau-silbernen Masse. Spàter nähert sich seine Farbe der des Kollagens (braun).

Was die kurzlich uber die Hyalinentartung gegebene Deutung an sich Wahrscheinliches hat, wage ich nicht zu entscheiden, weil noch ein sicherer Beweis erforderlich ist.

Wir haben nun die Verhaltungsweise des feinen Stutzgewebes bei dicser splenomegalischen Milz besprochen und es erubrigt noch, ennige Worte uber den Zustand der intraparenchymalen Gefaße mitzuteilen. Wir sagen vorerst, daß es eine hervorragende Gefäßneubildung und eine ausgreprägte Neubildung des perivaskulären Gewebes gibt. Alle neugebildeten Gefaße von mittelgroßem oder kleinem Durchmesser sind immer und dauernd von einem reichlichen elastischen Gewebe begleitet. Die Intima zeigt keine Proliferationserscheinungen und hat im allgemeinen das einschichtige Endothel immer gut erhalten. Unterhalb der Intima ist eine innere elastische Schicht recht deutlich und beständig vorhanden.

Bei den kleinen Gefaßen befinden sich außerdem eine schmale Adventitia und zwschen deren bindegewebigen Bundeln ganz düne elastische Fasern. Hicr ist das clastische Gewebe dem adventitialen Gewebe gegcnuber sicherlich uberwegend. Bei den Gefäßen, die einen gróßeren Durchmesser zeigen, ist dagegen das Bindegewebc im Vergleich mit den elastischen Elementen uberwicgend. Hier bildet die Adventitia einen wahren, sehr dichten, nicht sehr kernreichen Muff ohne Degenerationszeichen.

Die Muskelhaut ist gut entwickelt und ihre Tunika erreicht eine Dicke, die bei fast allen Gefäßen jener der Adventitia gleichkommt. Zwischen den emzelnen Fasern sind regelmaßig angelegte bindegewebige Streifen vorhanden.

Bei solchen Gefảßen ist auch das Ineinandergreifen der elastischen Fasern sehr gut entwickelt. Wo sie die innere elastische Haut bilden, sind die zahlreichen konzentrischen Fasern sehr dicht und deutlich, verdunnen sich aber und werden spälicher in der Muskelhaut, wenn sie auch die Anordnung zu fibrillaren Bündeln behalten.

In der Adventitia hören dagegen die elastischen Bündel auf und wir haben nur sehr schmale und reichliche Fibrillen, die unregelmäßig zwischen den bindegewebigen Bündeln zerstreut liegen. Auf mancher Stelle bilden diese sogar wahre Plexus, obglcich ihre Dunnheit eme ständige bleibt. 
An der Peripherie der Gefäße greift das Bindegewebe und das elastische Gewebe auf die Buchtungen und die Follikel über, besonders aber auf die ersteren. Sie tragen aber zur Verstärkung der Wand nicht bei.

Bei den Follikeln können wir jedoch - aber seltener - eine solche Verbreitung des elastischen Gewebes bemerken: es handelt sich dabei eben um jene Follikel, in welchen der Skleroseprozeß sich abwickelt oder beginnt. In diesem Falle dringen zwischen den Follikelelementen neben bindegewebigen Fasern noch elastische Fasern ein.

Letztere sind aber sehr spärlich, unregelmäßig zerstreut und sie befinden sich an der Peripherie der Follikel selbst, wobei sie sekundär zur Entstehung des Skleroseprozesses beitragen. Es ist sehr schwie. rig, unter dem Mikroskop die vollständige fibrös-elastische Sklerose eines Follikels zu erblicken. Meistenteils ist nur der peripherische Teil derselben (wenn es sich um eine extrafollikuläre Arterie handelt), der betroffen ist, wahrend die zentrale Zone meist verschont bleibt. Wenn wir aber mit Geduld untersuchen, dann finden wir manchmal bindegewebige regelmäBig rundliche Anhängsel, die cine normale, dem Follikel entsprechende Größe haben. Sie bestehen nur aus bindegewebigen, aufeinander liegenden und durch Schöpfe von elastischen Fibrillen durchschnittenen Bundeln und Fasern. Abgesehen von solchen Fällen, wo es sich um Teile der Gefaßadventitia handeln könnte, will ich diese Anhäufungen als in einer völligen Sklerosierung begriffene Follikel ansehen.

Was die Veränderungen der Milzvene anbelangt, wozu die ma$\mathrm{kr}$ oskopische Beschreibung am besten dient, werden wir noch einige Einzelheiten hinzufügen, die aus der histologischen Untersuchung sich ergeben.

Was die Dicke der Venenwände, die darin angetroffenen Veränderungen und die Ähnlichkeit mit dem Arteriengewebe betrifft, so haben wir das schon oben besprochen. Die splenische Vene wurde in der Nähe des Pankreasschwanzes untersucht, und zwar auf eincr Stelle, wo ihre Wand - obgleich verdickt - - weder Runzeligkeiten, noch Kalkerhöhungen noch Substanzverluste zeigte. An dieser Stelle war die Venenwand glatt und ununterbrochen, ganz frei von Endothel und aus einem fast hyalin entarteten Gewebe zusammengesetzt, welches sehr spärliche und stellenweise gar keine Zellelemente aufwies. Die Struktur dieser Zone ist eine deutlich bundelartig e mit parallelen Bündeln. In der Tiefe zeigen sich spärlich kapillare kleine Gefäße und einige glatte Muskelfasern. Die Dicke dieser Schicht ist eine ungleichmảBige. An der Stelle, wo dieselbe dünner wird, tritt die gut erhaltene und gut entwickelte Mittelhaut vor, welche oberflächlich reich an Muskelfasern ist und stellenweise von bindegewebigen Bündeln durchschnitten wird. Die Adventitia weist nichts Besonderes 
auf, mit Ausnahme einer starken Proliferation von Elementen, die sich hier und da zu Zentren vereinigen kơnnen. Die Entwicklung der Vasa vasorum ist eine ausgepragte.

Was das Verhalten der elastischen Fasern anlangt, bemerkt man die Bildung einer inneren elastischen Haut, die aber nicht eine fortlaufende, sondern stellonwerse eine unterbrochene und hin und wieder eine verdunnte ist.

Be1 der Mittelhaut ist das elastische Gewebe sehr sparlich vorhanden und nur an der Grenze der Adventitia lảßt sich eine äußere, gut entwickelte und ununterbrochene elastısche Haut erkennen. Selbst die Arterien sind von Veräderungen nicht fres und ich habe bei cinem arteriellen Zweige der splenischen Arterie (Hilus) von mittelmaßigem Durchmesser dic Unterbrechung und die Abwesenheit der inneren elastischen Haut auf einer langen Strecke beobachtet. Das Gefaßlumen ist dann won einer neuen, ebenfalls wellenformigen elastischen Haut umschrieben, die ich fur eme Neubildung halten möchte.

Zwischen beiden elastischen Platten befindet sich eine gewisse Anzahl von Kernen, die endothelialen Ursprungs sein konnten. In diesem Falle wurden dieselben für eine Proliferation der Intima sprechen. In den neugebildeten Vasa vasorum haben wir dagegen regelmaßige endotheliale Neubildungen.

Wir gehen nun zur Untersuchung der ibrigen Organe uber, die unsere Aufmerksamkeit auf sich gelenkt hatten. In dem $\mathrm{Pank}$ eas ist eine Neubildung von Bindegewebe, Gefaßen und Langerhansschen Herden in Masse vorhanden. Das immer von Gefaßen begleitete Bindegewebe - obgleich $c s$ in der Regel die Funktion hat, die Drusenherde zu begrenzen - ist hier durch dicke Bundel dargestellt, die jede einzelne Beere umgeben und absondern. Es sind nicht einmal die Langerhansschen Herde verschont, die im Gegrenteil durch dichte bindegewebige Streifen durchschnitten, durchfurcht und in mehrere Teile deutlich abgeteilt sind.

Die Neubildung von Bindegewebc ist bei den Ausfuhrungsgängen besonders offenbar. Letztere sind von einem wahren Muff umgeben, der neugebildete Gefảe enthalt. Die Gefaßße sind zahlreich und normal. Die Langerhansschen Inseln sind cbenfalls normal und so zahlreich, daß sie auf jedem mikroskopischen Fulde -- ausschließlich der rom Bindegewebe durchsetzten Herde - sofort in die Augen springen. Die Folge der bindegewebigen Proliferation ist natürlicherweise eine Sklerose. Wenn auch letztere in dem Pankreaskörper nicht viel bemerkt wird, ist sie dagegen im Schwanze, an der Stelle, wo wir die splenischen Gefaße untersucht haben (rom chirurgischen Präparate) am deutlichsten.

Ein solcher Skleroseprozeß macht sich naturlich mehr an den Acinis als an den Ausfihrungsgängen geltend, weil eben die ersteren von einer Hulle umgeben sind. Wir treffen in der Tat zahlreiche 
Beeren, die gänzlich durch den bindegewebigen Ring erwurgt werden und sich auf zwei oder drei noch färbbare Zellen beschränken. Bei anderen ist dagegen die Form einer Rohre noch erhalten, aber die Zellen sind klein, wenig gefärbt und auf dem, Wege, deutlich atrophisch zu werden. Dasselbe kommt in einem viel niedrigeren Grade bei den Langerhansschen Inseln wieder vor. Hier kann man wirklich nicht von einer bindegewebigen Substitution sprechen, sondern nur von einer Infiltration.

Bei dem post mortem entnommenen Pankreas sind außerdem noch neue Veranderungen vorhanden. In dem bei der Operation entnommenen Stucke sind die Gefäße normal. Bci dem Kadaverstuck bemerkt man tiefgehende Veränderungen. Selbst die mit einem mittelmäßigen Durchmesser versehenen Venen sind thrombosiert. Das Gefäß ist deutlich ausgedehnt und durch einen frischen Thrombus gänzlich verstopft. Dieser Thrombus besteht teils aus roten, teils aus weißen Blutelementen nebst Fibrinfäden, aber ohne Spuren von bindegewebiger Organisation oder Verflüssigung.

An der Venenwand zeigt sich das elastische Balkensystem normal. Außer diesen Venen, bei denen der Prozeß von Verstopfungsthrombose vollkommen ist, bemerken wir andere Venen, bei denen ein solcher Prozeß im Beginn steht. Diese Venen sind in der Nachbarschaft der oben erwähnten gelegen. Sie sind von reichlichem Bindegewebe umgeben, das sich in das intralobuläre Gewebe fortsetzt. Diese Venen sind ziemlich ausgedehnt und weisen Erscheinungen von frischer ausgeprägter Stauung auf. Die Innenwand dieser Gefäße ist normal. Obwohl es sich um Leichenpräparate handelt, ist die Intima noch erhalten. Die Arteriengefäße sind dagegen frei von Thrombosen.

In der Nähe der thrombosierten Venen zeigen die Pankreasacini Atrophiestellen. Sie haben namlich an Zahl abgenommen und sind sehr blaß gefärbt. Auf diesen Stellen fangen mit großer Wahrscheinlichkeit Entartungsprozesse an.

Gleichzeitig bemerkt man an fast allen Ausfuhrungsgängen eine Ausdehnung des Lumens und eine Lósung des Epithels. Nach meiner Meinung handelt es sich nicht um die gewòhnliche Ablösung, die man bei fixierenden Flüssigkeiten oder post mortem im allgemeinen bemerkt, weil man neben einer Ablosung noch einen deutlichen Entartungsproze $B$ der Epithelien beobachten kann. Letztere liegen nicht der Innenseite der Gänge an, wie wir es post mortem bemerken, sondern sie befinden sich teilwerse in dem zentralen Teile der ausgedehnten Röhren. Bei einigen Fällen könnte es sich um eine Ablösung handeln, weil die Epithelien noch gut erhalten und normal angeordnet sind. In anderen Fällen sind dagegen Entartungsveränderungen deutlich nachzuweisen. 
Zwischen den einzelnen Acinis bemerkt man ferner hier und da Fettinseln, was von der Fettentartung einer ganzen Beere oder von der Fettinfiltration des zwischen den Beeren liegenden Bindegewebes abhängen könnte.

In der Leber sind die Veranderungen noch schwerer und stehen im Zusammenhang mit dem verảnderten portalen Blutumlauf. Die makroskopischen Veránderungen der Vena porta und der intrahepatischen portalen Abzweigungen sind schon beschrieben worden.

Wir erwahnen hier nur, daß die beim Eintritt in die Leber untersuchte Vena porta beinahe dieselben Veränderungen wie die Milzvene aufwies.

Zunächst ist ihre Dicke je nach der Stelle verschieden. Wir aber haben uberall in einer mehr oder weniger hohen Schicht gegen das Lumen eine hohe bindegewebige, durch das Fuchsin stark rosa gefärbte Schicht, welche aus parallelen, aufeinander liegenden, an Zellelemonten sehr armen Bundeln besteht, die noch stellenweise, Desonders gegen die freie Flache, hyalin entartet sind. Bei dieser bindegewebigen Schicht fehlen gänzlıch die elastischen Fasern und auch die Gefäße sind darin sehr sparlich vorhanden. Es sind eben dunne Kapillaren, die von den hoher liegenden Schichten herstammen. Die Media ist wenig entwickelt, wahrend die Adventitia deutlich entwickelt, reich an bindegewebigen Bundeln und an elastischen Fasern ist, die eine regelmäßige Schicht bilden. Man findet darin noch glatte, eingeschaltete Muskelfasern.

Die Vasa vasorum sind sehr gut entwickelt und weisen in ihrer nächsten Umgebung noch leichte kleinzellige Infiltration auf. Was aber diese Gefaße besonders kennzeichnet, ist deren übermäßige Aus. dehnung durch dic Blutelemente. $Z_{\text {wischen dieser Adventitia und }}$ dem hepatischen Gewebc ist eine neue bindegewebige Schicht reichlich vorhanden mit den Eigenschaften des vorher beschriebenen Gewebes, jedoch viel reicher an Zellelementen und an Venen.

Manchmal findet man darin noch emige kleme Gallengänge.

Wir haben in der Leber zwischen den einzelnen Läppchen eine mäßige und gleichmaßige Ncubildung von Bindegewebe. Sie wird um die Gefaßc und die portalen Raume und auch um die Gallengefäße herum noch dichter. Die Läppchen sind recht deutlich, sei es wegen des oben erwähnten Bindegewebes oder wegen der mäßigen Ausdehnung der zentralen Vene, die gleichzeitig zur Orientierung dient.

Bei den kleinen portalen Gefäßen sind die Erscheinungen einer frischen, wellenartigen Thrombose schon deutlich und mit den Eigenschaften, dic wir beim Pankreas beschrieben haben.

Das hepatische Gewebe ist dort besonders verändert, wo es sich in Beriuhrung mit den großen portalen $Z_{w}$ eigen befindet. 
Von der äußeren Wand dieser letzteren schieben sich große bindegewebige Herde (worin die neugebildeten Gallengefäßchen hervortreten) zwischen den Läppchen ein, wobei sie eine deutliche Begrenzung zeigen. Diese bindegewebigen Herde wechseln außerdem mit Stellen von Atrophie und ausgeprägter Fettinfiltration ab. Weiter von den portalen Ästen weg werden diese Stellen allmählich kleiner und verschwinden sogar. Wir konnen also das cigentliche hepatische Gewebe - ausschließlich der schon besprochenen intralobulären bindegewebigen Verdickung, die aber nur mäßig stark ist - als normal ansehen. Hier und da sind auch intralobuläre Gallenkapillaren sichtbar, welche durch das Fuchsin rot gefärbt werden.

Was den Sitz der hepatischen Thrombosen betrifft, habe ich dieselben ausschließlich bei den portalen Räumen und bei den von diesen venösen Räumen nach der zentralen Vene gerichteten $Z_{\text {weigen an- }}$ getroffen. Bei den zentralen Venen, auf der Höhe des sogenannten großen venösen Blutumlaufs, habe ich aber nur stellenweise Erscheinungen von Stauung und niemals von Thrombose bemerkt. Der Thrombus ist überall neu entstanden und erreicht nie - wenigstens bei Venen mit einem mittleren Durchmesser - bindegewebige Organisation. Von gleichem Alter sind noch alle anderen Thromben auf der Höhe desselben portalen Raumes. Die Abzweigungen der hepatischen Vene zeigen nichts Besonderes. Auch die Gallengefäße sehen normal aus, obwohl sie von einem reichlichen bindegewebigen Ringe umgeben sind.

Auf den Darm und das Mesenterium beziehen sich endlich die letzten rückgängigen Veränderungen des portalen Systems. Dieselben sind durch die schon beschriebenen makroskopischen Gefäßveränderungen an der Insertionsstelle des Mesenteriums am Dünndarm und durch den roten Infarkt eines Teils des Dünndarms selbst gekennzeichnet. Die Veränderung der venösen Gefaße ist beim Mesenterium deutlicher als bei den ubrigen untersuchten Organen nachweisbar.

Wir haben hier an der Insertionsstelle des Mesenteriums am Dünndarm den arteriellen Zweig und den entsprechenden venösen. Die Arterie ist ganz normal, die Vene dagegen erweitert und durch ein frisches, anhängendes Gerinnsel verstopft. Ein Druck auf die Venenwand ist deutlich zu erkennen: die Wand zeigt sich dünn, mit spärlichen bindegewebigen und elastischen Fasern. Von der Intima ist keine Spur mehr vorhanden.

Richten wir unsere Aufmerksamkeit auf das Fettgewebe des Mesenteriums, so bemerken wir darin ganz frische Entzündungserscheinungen. Wir haben die ersten Andeutungen von den das Fettgewebe begrenzenden Kapillarräumen, worin sich Anhäufungen von Leukocyten befinden, zunächst nur an einigen Stellen, dann an mehreren, bis schließlich große und weite Zonen kleinzelliger Infiltration 
überall um bald thrombosierende Gefäße bilden. Auf Mikroorganismen habe ich die Schnitte nicht untersucht, weil es sich um eine laparotomierte Frau handelte, bci welcher wir schließlich einen ausgedehnten Infarkt des Dunndarms hatten. Das Vorhandensein von Mikroorganismen hàtte nichts Neues hinzugefugt.

Ich will jedoch noch bemerken, daß bei den untersuchten kleinzelligen Zentren und bei der Masse der Thromben niemals Anzeichen von Verflüssigung angetroffen wurden.

Wir haben nun die histologische Beschreibung aller anatomischen und chirurgischen Schnitte beendigt, die für unseren Fall ein besonderes Interesse hatten. Diese Beschreibung mag vielleicht hier und da zu ausführlich gewesen sein, uns schien es aber notwendig zur Erreichung der genauen Diagnose und zur Deutung der verschiedenartigen Abschnitte des Prozesses. Wir stellen uns zunächst folgende grundsätzliche Frage:

Handelt es sich wirklich um eine Bantische Krankheit in einem bestimmten Zeitraum ihrer Evolution? Auf den ersten Blick könnte es vielleicht scheinen, daß diese Frage nicht am Platze sei. Wenn wir uns aber an die makroskopischen Veränderungen der entfcrnten Milz und an den vollständigen Befund der Autopsie halten, so finden wir Tatsachen, die an der gefaßten Diagnose zweifeln lassen.

Banti hat im Jahre I 894 unter dem Namen, ,Splenomegalie unter hepatischer Cirrhose" ein spezielles Syndrom beschrieben, das viele gemeinschaftliche Eigenschaften - besonders im ersten Stadium - mit der splenischen Anämie der Erwachsenen zeigte, aber von dieser wegen des späteren klinischen Verlaufs sich dadurch unterschied, daß die erste sich nach der hepatischen Cirrhose abwickelt, während die zweite nie bis zu diesem Stadium kommt. Wenn wir nun die Krankengeschichte, die klinische Untersuchung usw. noch einmal durchgehen, können wir dann unseren Symptomenkomplex jenen anreihen, den Banti als bezeichnend für seine Krankheit beschrieb? Die Antwort ist sicherlich eine bejahende. Für das klinische Ganze, dessen Wiederholung und Behandlung hier unnötig ist, war die Diagnose auf Bantische Krankheit zwischen dem 2. und 3. Zeitraum diejenige, die am besten entsprach und sich außerdem auch durch Ausschließung als erforderlich erwies. 
Stimmt aber der anatomische Befund mit dem klinischen überein?

Hier beginnen gerade die Zweifel. Sie betreffen die makroskopischen wie auch die mikroskopischen Angaben.

Bei den ersteren tauchen $Z$ weifel auf über den in der chirurgisch entfernten Milz angetroffenen Infarkt, über die schon beschriebenen Veränderungen der Porta und der Splenica und über die mesenterialen Thrombosen nebst allen Folgen derselben.

Sind diese Veränderungen mit der Bantischen Splenomegalie in Einklang zu bringen? Um darauf eine Antwort zu zeben, brauchen wir eine möglichst große Anzahl von Fällen, die sehr gut beschrieben sind und über die noch Autopsiebefunde vorliegen. Durch Vergleich mit diesen Fällen wird es uns möglich sein, die Befunde unseres Falles zu deuten. Wir müssen jedoch notwendigerweise eine kritische Revision der die Bantische Krankheit betreffenden Statistik vornehmen, da wir natürlich alle Formen ausschließen müssen, die - obgleich sie eine Splenomegalie aufweisen - einen anderen ätiologischen Faktor für die Erklärung derselben erkennen lassen.

In seiner Abhandlung „Pathologische Anatomie“, und zwar bei der Beschreibung àer anatomischen Unterscheidungszeichen der seinen Namen tragenden Krankheit spricht Banti von splenischen Infarkten auch bei chirurgisch entfernten Milzen nicht. Bei der Besprechung der Veränderungen in der splenischen Vene schreibt er, daß nicht immer, aber häufig am Stamm der Vene die Intima stellenweise verdickt sei infolge chronischer sklerotisierender Endophlebitis mit mehr oder weniger zahlreichen und großen Herden.

Er fügt noch hinzu, daß es wahrscheinlich ist, daß auch am portalen Stamm, jenseits der Ausmündung der Splenica ähnliche Veränderungen bestehen, die aber in den Mesenterialvenen fehlen. In der dritten Periode weisen auch die intrahepatischen Zweige der Porta Anzeichen von sklerotisierender Endophlebitis auf, und auch die Mesenterialvenen könnten eine solche zeigen. All diese Veränderungen sind nun unter keinem Gesichtspunkte mit den von uns angetroffenen Veränderungen zu vergleichen.

Micheli, der fünf Fälle von Bantischer Krankheit mit und ohne hepatischer Cirrhose ausführlich untersucht hat, führt 
1n seinem 2. Fall an, daß die Innenfläche der splenischen Vene bei dem Hilus durch eine harte, weiße Sklerosisplatte gekennzeichnet war. In seinem 3. Fall beschreibt er einen weißlichen Fleck, der einen Durchmesser eines ital. ro-Cents-Stückes hatte, und das auf deren äußerem Milzrand zwischen oberem und mittlcrem Drittel gelegen war. Dieser Fleck vertiefte sich ins Parenchym und hatte die charakteristische Gestalt eines hämorrhagischen Infarktes. Die Hilusgefäße wiesen keine makroskopisch diagnostisch wichtige Strukturveränderungen auf. Die Porta war frei und ebenso auch die portalen Wurzeln. Der Darm war normal. (Die Patientin starb zwei Tage nach der Splenektomie.)

In seinem 4. Fall schreibt Micheli, daß die Hilusgefäße sehr weit waren, ohne jedoch makroskopische Veränderungen aufzuweisen. Mikroskopisch war eine Endophlebitis der Splenica vorhanden. Auch in seinem 5. Fall erwähnt der genannte Autor, daß die Hilusgefäße merkwürdige Veränderungen zeigten und daß auch der Stamm und die Hauptabzweigungen der Porta normal waren.

All diese Veränderungen sind mit denen nicht zu vergleichen, die wir bei dem splenischen Hilus, bei der splenischen Vene und bei der Vena porta und deren Verzweigungen bemerkt hatten.

Ich hàtte hier ein ganzes Literaturverzeichnis, wenigstens das italienische, über die Splenektomie bei Bantischer Krankheit anführen können, um Befunde zu suchen, die sich meinen nähern: eine solche Arbeit wurde aber von Micheli selbst ausgeführt, welcher bei der Besprechung der Auswahl - die wir nötigerweise vornehmen müssen, um von dem Bantischen Bilde oder von der splenischen Anamie nebst Splenomegalie alle anderen Syndrome zu beseitigen, welche ein anderes ätiologisches Moment erkennen lassen --, noch jene Fälle anführt, die im allgemeinen auf die splenische Anämie oder auf die Bantische Krankheit zurückgeführt werden, bei welchen so schwere und auf die Gegend der Vena porta verbreitete Veränderungen (chronische, adhäsive Phlebitis) vorhanden waren. Es ist rernünftiger, diese Veränderungen mehr als primäre anzusehen, wie als sekundäre im Sinne der von Banti bei der splenischen Anämie mit oder ohne Lebercirrhose beschriebenen speziellen Endophlebitis. Und diese Betrachtung ist um so einleuchtender - fügt Micheli noch hinzu 
- als die Veränderungen infolge Phlebitis nebst sekundärer Thrombose - obgleich auf der Splenica überwiegend - in mehr oder weniger breiter Weise andere portale Wurzeln betreffen, wie 5 von Micheli erwähnte Verfasser beobachtet haben. Letztere würden also die Fälle sein, die sich in bezug auf die Veränderungen des splenischen Hilus und des portalen Hilus am meisten meinem Falle nähern würden. Ich habe aber diese von Micheli erwähnten Fälle nicht durchlesen können, da dieselben in ausländischen Zeitschriften veröffentlicht wurden. Ich kann also nicht wissen, inwieweit mein Fall sich auf gewisse chronische Formen von Splenomegalie pylethrombotischer Entstehung zurückführen läßt, zu welchen Formen Micheli die 5 oben erwähnten Fällc zählt.

Wenn ich aber auch jene Fälle, die für mich ein großes Interesse gehabt hätten, nicht im Originale lesen konnte, habe ich doch - mittelbar oder unmittelbar - eine sehr große Anzahl von Splenektomien unter Augen gehabt, die wegen Bantischer Krankheit ausgeführt worden waren. Bei ganz wenigen wurde auf schwere Veränderungen der portalen-splenischen Gefảße hingewiesen.

Wir müssen uns aber an folgende Tatsachen erinnern: In der operativ entfernten Milz war schon ein anämischer Infarkt mit Erweiterung und Kalkinfiltration des Hilus vorhanden. Daß diese Erscheinung wenigstens seit einigen Jahren datiert, ist durch die Autopsie bewiesen, wobei wir sahen, daß die splenische Vene einen maximalen Durchmesser von $10 \mathrm{~cm}$ erreicht hatte und die stark ditalierte portale Vene einen Durchmesser von $8 \mathrm{~cm}$ zeigte. Beide haben die Struktur einer Arterie mit atheromatösen und kalkhaltigen Wänden.

Bestehen diese sıcherlich alten Veränderungen schon vorher oder sind sie der Splenomegalie gefolgt?

Obwohl ich eine gewisse Erfahrung bei der Deutung der in den verschiedenen Organen aufeinander folgenden Veränderungen habe, so bin ich doch nicht in der Lage, eine einwandfreie und befriedigende Antwort darauf zu geben.

Wenn jemand behaupten würde, daß die primäre Veränderung ihren Ursprung in der Vene gehabt hat, könnte ich ihm einwenden, daß die bei der splenomegalischen Milz angetroffenen 
Veränderungen nicht denen entsprechen, die eine solche Milz hätte haben sollen, bei welcher wegen eines chronischen Gefäßprozesses der Rücklauf behindert war.

Es fehlen gänzlich die Charaktere der frischen Stauung und das ist leicht begreiflich. Es fehlen aber auch die Kennzeichen einer chronischen Stauung. Obwohl wir wissen, daß auf jede chronische Stauung eine bindegewebige Neubildung folgt, kann man sicherlich nicht die Charaktere einer verbreiteten, bindegewebigen, schon bei dem makroskopischen Befunde deutlich ersichtlichen Hyperplasic - nach meiner Meinung - als ein Endstadium enner Stauung mit langem Verlauf auffassen. Wenn wir auch dıe Gefäßveränderung als primär ansehen wollten, fehlte uns jedoch jede Verbindung zwischen der Gefäßveränderung und der Splenomegalie. Wenn wir dagegen die letztere als primäre annehmen wollten, so würden die bei den Gefäßvenen angetroffenen Veränderungen unerklärt bleiben.

Bei der splenomegalischen Milz der Bantischen Krankheit ist die sklerotische Endophlebitis der Splenica und auch der Porta ein Prozeß, welcher, mit bloßen Augen gesehen, sich höchstens als eine einfache Verdickung der Innenwand ergibt, aber er erreicht nie die Schwere und die Bedeutung des von mir angetroffenen Prozesses. Ich kann also über die Verhältnisse zwischen den angetroffenen Veränderungen und der Splenomegalie kein Licht bringen, wie auch nicht einmal annähernd die Stelle bestimmen, wo der Prozeß seine Entstehung gehabt hat, ob in der Vene oder in der Milz.

Wollte man also festhalten, daß das Vorhandensein der beschriebenen venösen Veränderungen, die sich zu einer Splenomegalie gesellen, die Deutung dieser letzteren überaus erschwert, dann fragen wir, ob durch die makroskopischen Befunde der Splenomegalie es moglich ist, die klinisch schon gefaßte Diagnose aufrecht zu erhalten. Ohne jetzt hier diese Befunde wiederholen zu wollen, genügt uns die Schlußfolgerung, daß dieselben im allgemeinen mit denjenigen übereinstimmen, die $\mathrm{Banti}$ für seine Krankheit beschrieben hat.

Die Veränderung beschränkt sich auf eine Verdickung des Netzgewebes und diese Verdickung ist von B a nti mit dem Namen „Fibroadenie" bezeichnet worden. Der Prozeß beginnt gewöhn- 
lich in der Nachbarschaft der Follikelarterie und wir haben die ganze Evolution des sklerotischen Prozesses bis zu seiner vollständigen fibrösen Umwandlung verfolgt. Auch die extrafollikulären Arterien zeigten einen ähnlichen Prozeß.

In der Pulpa fehlten die Kennzeichen der Stauung, obwohl in der Leber schon bindegewebige interlobuläre Verdickungserscheinungen vorhanden waren. Auch hier war die Entwicklung der Stränge einer Verdickung des Stützgewebes zuzuschreiben. Man hat dabei weder eine Siderosis noch pigment-, noch globulihaltige Zellen wahrgenommen, wie es bei einer chronischen Stauung der Fall ist. Auf dem Komplex dieser Veränderungen - zu denen man noch die splenische Endophlebitis zählen muß - gründet sich die Diagnose der Bantischen Krankheit. Wir wissen jedoch, daß dieser Prozel3 von „Fibroadenie“ keineswegs für diese Krankheit bezeichnend ist; er ist aber ein Merkmal derselben.

Dieser Prozeß ist von zahlreichen Untersuchern bei Splenomegalien verschiedener Entstehung, und zwar in Form von Leukämie, Pseudoleukämie usw. und sogar in den Milzen bei der gewöhnlichen Lebercirrhose (Micheli) bemerkt worden.

Deswegen kann man die Splenomegalie nicht als ein spezifisches Reaktionsprodukt gegen einen bestimmten krankhaften Anreiz, sondern vielmehr als die Folge eines Prozesses von bindegewebiger Sklerose nach chronischen Reizen verschiedenen Ursprungs ansehen.

Bevor ich diese Arbeit schließe, will ich noch einige Betrachtungen in bezug auf die Befunde der Autopsie beifügen. Die pathologisch-anatomische, vom Prof. Guizzetti gestellte Diagnose war folgende:

Vielfache Thrombosen nebst Phlebosklerose der Splenica, der Porta und mesenterische Thrombosen. Hämorrhagischer Infarkt des Dünndarms. Allgemeine Anämie nach Splenektomie wegen Bantischer Krankheit.

Es genügt hier, den in der Milz bei der Operation angetroffenen Infarkt und die Kreislaufstörungen zu erwähnen, welche die Folge der beschriebenen Veränderungen der Porta und der Splenica sein mußten, um mit Sicherheit als Schlußergebnis dieser Veränderungen die vielfachen Thrombosen annehmen zu können, die bei den verschiedenen Organen (Leber, Pankreas und Darm) 
angetrofferı wurden. Die Erkrankung des Darmes war sehr bezeichnend, weil es sich um einen roten hämorrhagischen Infarkt handelte, der $50 \mathrm{~cm}$ unterhalb des Duodenum begann und sich gegen den Blinddarm auf eine über I $m$ lange Strecke fortsetzte. Diese verschiedenen thrombotischen Prozesse, welche alle einen Verschluß der Venen herbeiführten und unter denen der den Infarkt des Dünndarms hervorgerufene der schwerste und deswegen die direkte Todesursache war, sind nur die Fortsetzung von dem, was wir an der Milz schon bei der Operation bemerkten, wo sich ein anämischer Infarkt vorfand.

Der Thrombosierungsprozeß 3 hat nämlich zuerst die splenische Vene und dann die Porta getroffen, wobei er in der Leber sehr beschränkte Veränderungen vermöge des darin vorhandenen doppelten Kreislaufes verursachte und zuletzt hat dieser Prozeß absteigend die Wurzeln der Porta selbst getroffen, indem er zum Darminfarkt Veranlassung gab.

Die Operation hat auf die Entwicklung dieser Thrombose wegen folgender Grunde nicht einwirken können: Wir haben auf dem chirurgischen Präparat einen sich auf dem Bildungswege befindlichen Infarkt gefunden, und das gibt an, daß die Umlaufsstorung in dieser splenomegalischen Milz schon vorher bestanden und daß in derselben der Rücklauf durch die Erkrankung der Splenica gehindert war.

Auf gleiche Weise, wie man diesen Infarkt in der Milz erklärt, erklärt man auch die im Darm angetroffenen Infarkte, während in der Leber die portale Thrombose nicht imstande war, den Infarkt zu erzeugen, weil die hepatische Arterie normal blieb.

Bei der Unterbrechung des arteriellen und venösen Kreislaufs wird die Splenektomie nicht anders wirken, als den Druck in den anderen Organen zu erhöhen, was die Thromboseerzeugung mehr hindern als begünstigen kann. Die wahrgenommenen Veränderungen bestanden also schon bei der Operation und sie können durchaus nicht auf diese zurückgeführt werden.

Ziemlich selten ist dagegen die venöse rücklaufende Thrombose mit darauffolgendem hämorrhagischen Infarkt zu betrachten.

Auf Grund der Littenschen Erfahrungen ist die Verhaltungs. weise der oberen Mesenterialarterien einer Unterbindung gegenüber allen bekannt. Diese anatomisch anastomosierte Arterie ist 
unter dem funktionellen Gesichtspunkte eine endanastomosierte Arterie und deswegen gibt uns die Unterbindung derselben einen klassischen Infarkt. Diese Tatsache, durch Versuche erhärtet, bedarf keiner Bestätigung.

Ganz neu sind dagegen unsere Kenntnisse über die Folgen der Verstopfung der Mesenterialvenen. Noch im Jahre 1898 haben Nothnagel und Mikulicz angenommen, daß die Verstopfung der Venen die gleichen Folgen wie die der Arterien habe.

Die Thrombose der Mesenterialvenen kann primär oder sekundär sein. Unser Fall gehört zu der zweiten Form. Bei den wenigen anderen beschriebenen Fällen wird die Ursache dieser Thrombose gesucht in den Leberveränderungen nebst nachfolgender Thrombose der Porta, so in Cirrhose, Syphylis, Geschwülsten, Fettentartung der Leber usw. Noch häufiger kommt die primäre venöse Mesenterialthrombose vor. Während die sekundäre nur eine abdominale Entstehung hat, kann die primäre auch außerhalb des Bauches ihren Ursprung nehmen. Tatsächlich erwähnt $Z$ esas drei Fälle von venöser Thrombose mit nachfolgendem Infarkt, wovon der eine auf eitrige Parotitis, der zweite auf eine erysipelatöse Hirnphlegmone und der dritte auf eine partielle Strumektomie folgte.

Die primären Thrombosen abdominalen Ursprungs stellen natürlich die Mehrheit dieser Fälle dar. Sie nehmen meistens ihren Ursprung in infektiösen Wochenprozessen, Früh- oder Fehlgeburten, Krebs des Rektums. Sprengel beschrieb einen Fall, der auf einen prostatischen Gonokokkenabszeß 3 folgte. Die gewöhnliche Ursache für diese Thrombosen liegt aber in den Darmaffektionen. Wir erwähnen Appendicitis, inkarzerierte Hernien, die Operationen an Hernien, Magen und Milz. Der Fall von Delatour (von Zesas angeführt) ist interessant: „Eine wegen Splenomegalie operierte Person, die 23 Tage nach der Operation akute Baucherscheinungen bekam, starb innerhalb 24 Stunden. Bei der Autopsie fand man einen hämorrhagischen Infarkt, der den dritten Teil des Dünndarms einnahm, nebst einer Thrombose der Splenica und der beiden Mesenterialvenen." Dieser Fall hat Ähnlichkeit mit meinem, jedoch besteht der Unterschied, daß es sich bei ihm wahrscheinlich um einen infektiösen, postoperativen, 
absteigenden Prozeß handelte, während in meinem Falle schon bei der Operation Kreislaufstörungen nebst deren Folgen vorhanden waren.

Man hat auch Thrombosen als Folgen nach abdominalen, syphylitischen, tuberkulösen, typhösen Geschwüren usw. beobachtet. In bezug auf die Stelle, die mit Vorliebe von dem Infarkt nach venösem Verschluß getroffen wird, stimmt mein Fall mit den anderen ganz überein. Im allgemeinen wird der Anfangsteil viel seltener als der mittlere Teil und nur ausnahmsweise der Endteil des Dünndarms getroffen. Eine Ausnahme ist die Thrombose der unteren Mesenterialvene. Auf diese folgt nicht immer der Infarkt wegen der größeren Anzahl von Anastomosen, die zwischen dieser Vene und der Cava vorkommen.

Der Symptomenkomplex der venosen Thrombose ist verschieden, je nachdem sie primär oder sekundär ist.

Die Symptome zeigen sich in einer akuten Form bei primärer Thrombose. Bei sekundärer Thrombose gehen Erscheinungen voraus, die auf ein Hindernis in dem portalen Kreislaufe hindeuten, wie: diffuse Bauchschmerzen, Durchfall, Ascites usw. Blutige Entleerungen sollen aber kein beständiges Symptom sein. Sie sind kennzeichnend, wenn sie vorhanden, können aber auch ganz fehlen. Unter I 53 von Jackson untersuchten Fällen (in der Zesasschen Arbeit angeführt) waren blutige Stühle nur in I9 Proz. angegeben. Unsere Patientin hatte dieselben nur in den letzten 24 Stunden, aber viel häufiger Neigung zum Erbrechen und andauernde Durchfälle (auch vor der Operation).

Man hat auch zwei klinische Formen von venöser Thrombose aufgestellt, wobei man sich auf folgende Erscheinungen stützen könnte : Bei der ersten Form überwiegen der Durchfall, die Darmblutungen, das Brechen usw. und der Tod tritt zwischen 24 und 48 Stunden ein. Bei der zweiten Form ist das Bild des paralytischen Ileus mit Abwesenheit ron Hämorrhagien überwiegend, und der Tod erfolgt zwischen 5-6 Tagen.

Es ist für alle Verfasser immer eine schwere Sache gewesen, eine passende Erklärung zu geben für beide Formen der Erkrankung. Nur Sprengel (s. Zesas) nimmt zwei verschiedene anatomische Veränderungen an: in dem einen Falle hatte man es mit einem hämorrhagischen Infarkt $z u$ tun und in dem anderen 
mit einem anämischen Brande. Bei dieser Unterscheidung nimmt aber $\mathrm{Sprengel}$ an, daß der erste Fall immer einem venösen oder arteriellen Verschlusse seine Entstehung verdankt. Den zweiten Fall hätte man nur bei Verstopfungen arteriellen Ursprungs, wenn der Blutrücklauf fehlt. Diese Einschränkung lenkt uns vom Gebiete der venösen Thrombosen ab und soll deswegen hier nicht erörtert werden. Klinisch bestehen sicher beide Formen. Aber alle kennen die großen Schwierigkeiten, die venösen von den arteriellen Verstopfungen zu unterscheiden und zu begrenzen, um so mehr, als noch dazukommen die seltenen Fälle, bei denen die Verstopfung sich gleichzeitig auf die Venen und auf die Arterien erstreckt. Es ist also wahrscheinlich, daß die klinisch aufgestellte Unterscheidung in bezug auf die Thrombosen schwer eine Bestätigung in dem anatomischen Befunde finden wird. Andererseits muß der anämische Brand noch besser untersucht werden.

Meyer (in dem Journal der Chirurgie - Februar I9I2 angeführt) beschreibt einen Fall von anämischem Infarkt des Dünndarms :

Es handelte sich um einen 70 Jahre alten Mann, der ins Krankenhaus wegen allgemeiner Schwäche und Gliederbeschwerden aufgenommen wurde. Diese Schmerzen hatten vor 4 Wochen begonnen. Patient zeigte außerdem noch Alterskachexie, Arteriosklerose und Emphysem. In den ersten ro Tagen seines Aufenthalts im Hospital hatte er fortlaufend Fieber. Dann verschwand es, der Kranke wurde schwächer und starb am 38. Tage nach seiner Aufnahme, ohne die geringsten Darmstörungen gezeigt zu haben.

Bei der Autopsie fand man, was den Darm betrifft, folgendes:

Die Dünndarmschlingen liegen im kleinen Becken, sehr leicht adhärent. Jedoch ist eine Darmschlinge $\mathrm{I}, 50 \mathrm{~m}$ von der Ileocöcalklappe entfernt, mit fibrinösen Exsudaten und mit einigen kleinen Gerinnseln bedeckt, reichlich vaskularisiert. An dieser Stelle ist die Darmschlinge hart, auf einer $4-5 \mathrm{~cm}$ langen Strecke weiß-gelblich, an ihren Enden durch einen hämorrhagischen Ring deutlich begrenzt.

Das zuführende Segment ist erweitert, das abführende verengert. Nachdem die Darmschlinge geöffnet war, sah man, daß die Schleimhaut geschwollen und gallig verfärbt war. Die Schleimhautfalten waren mit Ausnahme der Stelle der Mesenterialinsertion verschwunden. Diese Schleimhaut ist von den benachbarten unveränderten Teilen durch zwei kreisförmige Geschwüre getrennt. Diese laufen rings um den ganzen Darm und sind $2-3 \mathrm{~cm}$ breit. Das ne- 
krotisierte Segment mißt $4 \mathrm{~cm}$ und hier ist der Darm doppelt so dick wie normal. An der Insertionsstelle des Mesenteriums befindet sich eine zylindrische Verdickung. Auf dem Schnitt erkennt man eine blumenkohlartige, gelbliche, aus nekrotisiertem Gewebe und kleinen arteriellen, sklerotisierten Gefäßen bestehende Masse. Diese Gefảße weisen ein verschlossenes Lumen auf.

Aus der histologischen Untersuchung ergab sich auf der Höhe des Infarktes eine vollständige Nekrose der Schleimhaut und der Submucosa. Bei den Geschwuren sah man erweiterte Kapillaren. nebst Herden von kleinzelliger Infiltration. An der Insertionsstelle des Mesenteriums ist eine starke Infiltration von Leukocyten vorhanden. Die Kapillaren und die kleinsten Venen sind mit Blut gefullt und teilweise thrombosiert. Das Lumen der Arterien ist gänzlich verschwunden, sei es infolge der Proliferation des Bindegewebes oder der neuen Thromben. Nach der Meinung des Verfassers handelte es sich um einen typischen anämischen Infarkt. Dabei waren die Arterien infolge einer chronischen, alten, verstopfenden Endoarteriitis verändert. Es schloß sich noch eine akute Thrombose an, welche sich ebenfalls auf die Venen verbreitet hatte.

Obwohl diese meine Untersuchungen nicht $\mathrm{zu}$ einwandfreien Resultater führen konnten, habe ich doch nach der Anamnese, nach dem klinischen Verlaufe und nach den histologischen Veränderungen der Milz auf eine primäre Veränderung der Milz schließen müssen mit den Kennzeichen der splenischen Fibroadenie. $\mathrm{Zu}$ dieser gesellte sich später noch eine schwere Veränderung der portalen-splenischen Gefäße, wahrscheinlich durch das Agens erzeugt, das auch die Splenomegalie hervorgerufen hatte.

Es kam zu Kreislaufstörungen infolge dieser Veränderungen und nicht etwa infolge entzündlicher Erscheinungen (Pylephlebitis). Als einfach hydraulisch erklärt sich die sekundäre rückläufige Thrombose in einigen Mesenterialvenen und endlich der Infarkt. Dieser war die Ursache des Todes.

Das folgende Literaturverzeichnis macht auf Vollständigkeit keinen Anspruch.

\section{Literaturverzeichnis.}

I. B a rbacci, Il fegato duro arteriosclerotico. Lo Sperimentale igro.

2. Herxheımer, Zur Pathologie der Gitterfasern der Leber. Zentr. allg. Path. 1907, Bd. I8. Verhandlungen der Deutschen pathologischen Gesellschaft, II. Jahrg. Jena, Fischer, 1908. 
3. Maresch, Über Gitterfasern der Leber und die Verwendbarkeit der Methode Bielschowskys zur Darstellung feinster Bindegewebsfibrillen. Zentr. allg. Path. I905, Bd. I6.

4. Micheli, Sul morbo di Banti. Archivio per le Scienze Mediche I909, Bd. 33 .

5. Rössle, Über die Metaplasie von Gitterfasern bei wahrer Hypertrophie der Leber. Verhandl. der Deutschen pathol. Gesellschaft, I2. Jahrg. Jena, Fischer, 1908.

6. Rössle u. Joschida, Das Gitterfasergerüst der Lymphdrüsen unter normalen und pathologischen Verhältnissen. Zieglers Beiträge 1909, Bd. 45 .

7. Russahoff, Über die Gitterfasern der Lunge unter normalen und pathologischen Verhältnissen. Zieglers Beiträge 1909, Bd. 45.

8. Schmidt, Über die Stützsubstanz der Leber in normalem und pathologischem Zustande. Zieglers Beiträge 1908, Bd. 42.

9. Po m m e r, Il reperto anatomo-microscopico nell' infarto intestinale. Virch. Arch. rgro, Bd. 200.

10. Lesas, La trombosi e l'embolia dei vasi mesenterici. Zentralbl. für die Grenzgebiete 1910, Bd. I3. 